J. Mietzner, P. A. Hoeher, and M. Sandell, "Compatible improvement of the GSM/EDGE system by means of space-time coding techniques," IEEE Transactions on Wireless Communications, vol. 2, pp. 690-702, July 2003.

(C) 2003 IEEE. Personal use of this material is permitted. However, permission to reprint/ republish this material for advertising or promotional purposes or for creating new collective works for resale or redistribution to servers or lists, or to reuse any copyrighted component of this work in other works must be obtained from the IEEE. 


\title{
Compatible Improvement of the GSM/EDGE System by Means of Space-Time Coding Techniques
}

\author{
Jan Mietzner, Student Member, IEEE, Peter A. Hoeher, Senior Member, IEEE, and \\ Magnus Sandell, Associate Member, IEEE
}

\begin{abstract}
On the basis of the GSM/EDGE system, the application of space-time coding techniques is investigated, especially with regard to aspects of compatibility with current specifications. In this paper, simple special cases of space-time trellis codes and space-time block codes are considered, namely the delay-diversity and the Alamouti scheme. The performance improvements obtainable by means of these techniques in the case of a typical urban (TU) wireless channel are demonstrated both on the basis of analytical and simulation results. For the delay-diversity scheme, a lower bound on the bit-error probability is derived and an optimization of the intrinsic delay parameter is considered. For the Alamouti scheme, a novel trellis-based soft-output equalization and detection algorithm is presented, which is of same complexity as in the single transmit antenna case. Moreover, in the case of the Alamouti scheme, appropriate training sequence pairs are required. In this context, optimized partner sequences with respect to the eight GSM sequences are introduced yielding pairs that significantly outperform any pair of GSM sequences. Furthermore, the sensitivity of the delay-diversity and the Alamouti scheme to a time-variant TU channel is addressed as well as to nonperfect knowledge of the channel coefficients at the receiver.
\end{abstract}

Index Terms-Alamouti scheme, channel estimation (CE), delay diversity, equalizers, fading channels, GSM/EDGE system, performance analysis, RAKE receiver bound (RRB), space-time codes (STCs), training sequences (TSs), wireless communication.

\section{INTRODUCTION}

$\mathbf{T}$ HE APPLICATION of multiple transmit antenna techniques in wireless communications systems has recently gained much interest. It introduces an additional spatial component to the signal processing carried out in the transmitter and offers many possibilities of performance improvements upon systems employing only a single transmit antenna.

Apart from the Bell Laboratories layered space-time architecture introduced by Foschini in [1], where multiple transmit and receive antennas are utilized in order to accomplish higher throughput of data, space-time trellis codes (STTCs) [2]-[5] and space-time block codes (STBCs) [6], [7] are subject to current research activities.

STTC and STBC exploit spatial diversity yielding an additional diversity and/or coding gain and, thus, an improved

Manuscript received April 16, 2002; revised August 12, 2002; accepted November 22, 2002. The editor coordinating the review of this paper and approving it for publication is $\mathrm{H}$. Boelcskei. This work was supported in part by Lucent Technologies, Bell Laboratories U.K., Global Wireless Systems Research, Swindon, U.K.

J. Mietzner and P. A. Hoeher are with the Information and Coding Theory Lab (ICT), Faculty of Engineering, University of Kiel, D-24143 Kiel, Germany (jm@tf.uni-kiel.de; ph@tf.uni-kiel.de).

M. Sandell is with the Telecommunications Research Laboratory, Toshiba Research Europe, Bristol BS1 4ND, U.K. (e-mail: Magnus.Sandell@toshibatrel.com).

Digital Object Identifier 10.1109/TWC.2003.814349 bit-error performance compared with a single transmit antenna system. With STTC and STBC, multiple antennas at the receiver are optional. Spatial diversity results from the fact that the individual transmission paths from the transmit antennas to the receive antenna(s) are likely to fade independently; i.e., the probability that each path is degraded at the same time is significantly smaller than the probability that a single transmission path is in a deep fade.

The application of STTC and STBC in future wireless communications systems promises reliable transmission of high data rates, e.g., required for third-generation $(3 G)$ bearer services. Such services are usually characterized by asymmetric data traffic, where the predominant part of data transfer occurs in the downlink (DL) direction. A single user at a mobile station (MS) might download comparably large amounts of data from the base transceiver station (BTS), whereas rather little data traffic is required in the uplink in order to initiate the download. Therefore, in order to enhance the crucial DL direction, the application of STTC and STBC is very attractive because of optional multiple antennas in the receiver. By this means solely the BTS needs to be equipped with additional antennas (besides, multiple antennas are already employed by many BTS in current second-generation networks).

This paper considers the application of the delay-diversity [8], [9] and the Alamouti scheme [10] in a GSM/EDGE system (enhanced data rates for GSM evolution) [11]. The delay-diversity scheme and the Alamouti scheme are the simplest special cases of an STTC and an STBC, respectively.

Original aspects of this paper include transmitter structures designed with regard to compatibility aspects (Section II). For the case of the Alamouti scheme, appropriate pairs of training sequences (TSs) are introduced (Section III). The proposed pairs consist of an original GSM sequence and an optimized partner sequence. They perform significantly better than any pair of GSM sequences. A novel equalization and detection algorithm for the Alamouti scheme is presented (Section III), which is derived from the conventional max-log maximum a posteriori (MAP) algorithm for a single transmit antenna system [12] and is characterized by the same complexity. For related work, see [13]-[20]. New analytical results concerning the performance of the delay-diversity and the Alamouti scheme in wireless communication systems are given in Section IV. First, an improved version of the so-called RAKE receiver bound (RRB) [21, Ch. 14.5] is derived for the delay-diversity scheme. By means of this lower bound on the bit-error probability, it is possible to optimize of the intrinsic delay parameter of the delay-diversity scheme. For related work refer to [22]. Second, the sensitivity of the 


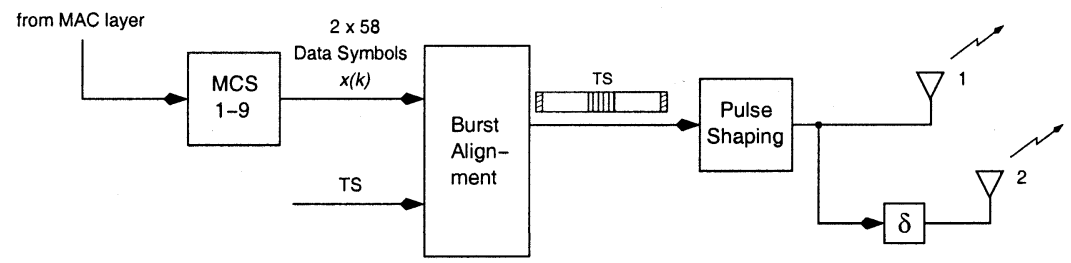

a)

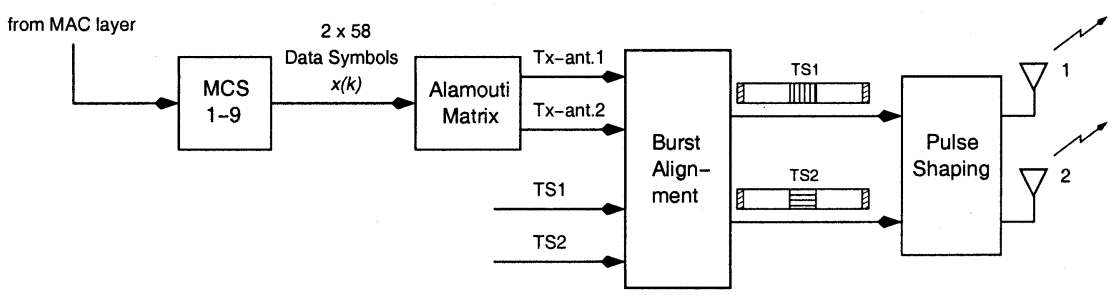

b)

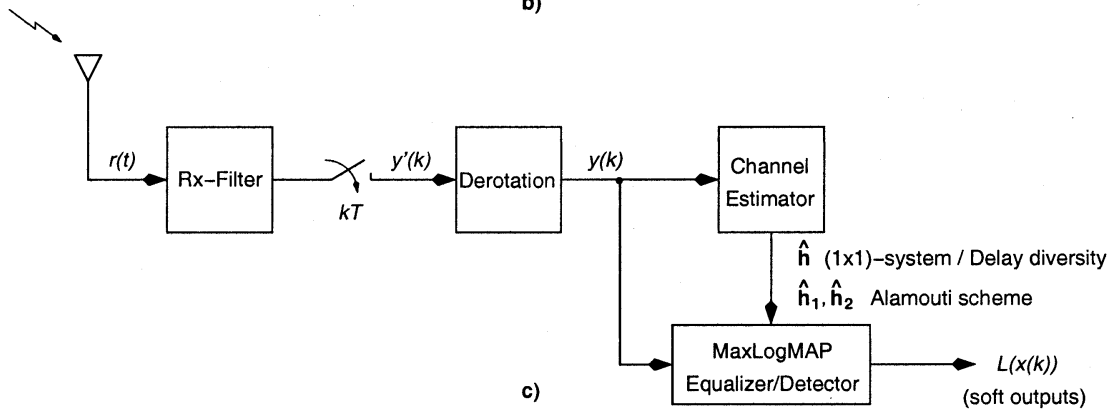

Fig. 1. Transmitter structure of the GSM/EDGE system enhanced (a) by delay-diversity $\left(n_{T}=2\right)$ and (b) by the Alamouti scheme $\left(n_{T}=2\right)$. (c) General receiver structure $\left(n_{R}=1\right)$.

Alamouti scheme to a fast-fading and to a frequency-selective fading channel is considered as well as to nonperfect knowledge of the channel coefficients at the receiver. Simulation results for the enhanced GSM/EDGE systems are presented in Section $\mathrm{V}$, demonstrating the bit-error performance improvements obtainable by either space-time coding (STC) technique in the case of a typical urban (TU) wireless channel. In particular, the influence of a time-variant channel and of nonperfect knowledge of the channel coefficients in the receiver is pointed out. Finally, a summary and concluding remarks are given in Section VI.

\section{Structure OF ThE ENHANCED Systems}

The compatible enhancement of the GSM/EDGE system by means of the delay-diversity and the Alamouti scheme, respectively, shall be carried out in a way that only a few changes have to be applied to current specifications [11]. In this context, the GSM/EDGE modulation schemes and the burst structure are retained in the extended systems. The EDGE specifications comprise both binary Gaussian minimum-shift keying (GMSK) and linearized GMSK with an eight phase-shift keying (PSK) mapping. Throughout this paper, the equivalent complex baseband notation is used.

\section{A. Transmitter Structure of the Delay-Diversity Scheme}

In the delay-diversity scheme, the same signal is transmitted over $n_{T}$ antennas applying different delays $\delta_{i}$ at each antenna $1 \leq i \leq n_{T}$. In the case that these delays are chosen as $\delta_{i}=$ $(i-1) T$ [9], where $T$ denotes the symbol interval, delay diversity can be regarded as the simplest special case of an STTC.
The transmitter structure of the GSM/EDGE system enhanced by delay diversity for $n_{T}=2$ is depicted in Fig. 1(a). First, channel coding, interleaving, and GMSK or 8-PSK mapping is performed according to the EDGE modulation and coding schemes MCS 1-9. This yields $2 \cdot 58 M$-ary data symbols $x(k)$ per burst, where $M=2$ for GMSK and $M=8$ for 8-PSK. Together with a TS-one of the eight GSM sequences as stated in Table $\mathrm{I}(\mathrm{A})$ - the $x(k)$ are mapped on GSM/EDGE bursts. Finally, linearized GMSK pulse shaping is done and the modulated signal is transmitted over two antennas, where a delay $\delta$ is applied at the second transmit antenna. Note that the single transmit antenna case $[(1 \times 1)$ system] is included in the enhanced structure as the special case, when the second transmit antenna is switched off. The enhanced system is, therefore, compatible with the $(1 \times 1)$ system and is characterized by the same data rate.

\section{B. Transmitter Structure of the Alamouti Scheme}

The Alamouti scheme is the simplest special case of an STBC and employs $n_{T}=2$ transmit antennas. Pairs of $M$-ary data symbols $(x(k), x(k+1))$ are transmitted over the two antennas each time using two consecutive time instants. In this context, a mapping of the symbols $x(k)$ and $x(k+1)$ is performed according to the Alamouti matrix $\mathbf{B}$

$$
\begin{array}{cc}
\mathbf{B}=\left[\begin{array}{cc}
x(k) & -x^{*}(k+1) \\
x(k+1) & x^{*}(k)
\end{array}\right] \leftarrow \text { Time index } k \\
\uparrow & \uparrow \\
\text { Ant. } 1 & \text { Ant. } 2 .
\end{array}
$$


TABLE I

(A) Eight GSM Training Sequences (Binary Representation) (B) Optimized Partner Sequences With Respect to the EIGHT GSM TRAINING SEQUENCES

\begin{tabular}{|c|c|c|c|c|c|}
\hline GSM TS No. & $\mathbf{A}$ & $\mathbf{B}$ & $\mathbf{C}$ & $\mathbf{A}$ & $\bar{B}$ \\
\hline 1 & $\begin{array}{lllll}0 & 0 & 1 & 0 & 0\end{array}$ & $\begin{array}{llllll} & 0 & 1 & 1 & 1\end{array}$ & $\begin{array}{lllllll}0 & 0 & 0 & 0 & 1 & 0\end{array}$ & 0001000 & 1001111 \\
\hline 2 & 000101 & $\begin{array}{lllll} & 0 & 1 & 1 & 1\end{array}$ & 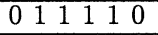 & $\begin{array}{llllll}0 & 0 & 1 & 0 & 1\end{array}$ & 10111 \\
\hline 3 & 010000 & 0101110 & $\begin{array}{lllllll}1 & 1 & 1 & 0 & 1 & 0\end{array}$ & 01010000 & 011110 \\
\hline 4 & 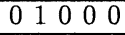 & 11110 & 110100 & 010000 & 11110 \\
\hline 5 & $\begin{array}{llllll}0 & 0 & 0 & 1 & 1\end{array}$ & $\begin{array}{llllll}0 & 1 & 0 & 1 & 1\end{array}$ & 1000100 & $\begin{array}{llllll}0 & 0 & 0 & 1 & 1\end{array}$ & $\begin{array}{lllll}0 & 1 & 0 & 1 & 1 \\
\end{array}$ \\
\hline 6 & 0101001 & 11010 & 110000 & $\begin{array}{llllll}0 & 1 & 0 & 0 & 1\end{array}$ & 11010 \\
\hline 7 & 10100 & 111111 & 0111000 & 10100 & 111111 \\
\hline 8 & 11101 & 11100 & $\begin{array}{lllllllll}0 & 1 & 0 & 0 & 1 & 0\end{array}$ & 11101 & 11100 \\
\hline $\begin{array}{c}\text { Partner Sequence } \\
\text { to GSM TS No. }\end{array}$ & $\mathbf{A}$ & B & C & $\mathbf{A}$ & B \\
\hline 1 & 00100 & 010001 & $\begin{array}{lllllllll}0 & 1 & 1 & 1 & 0 & 0 \\
\end{array}$ & 000100 & 01001 \\
\hline 2 & 0000011 & 10100 & 10000010 & 0000011 & 10100 \\
\hline 3 & $0 \begin{array}{lllll}0 & 1 & 0 & 1 & 1\end{array}$ & 01111 & $\begin{array}{lllllll}0 & 0 & 0 & 1 & 0 & 0\end{array}$ & 0101011 & 011111 \\
\hline 4 & 10111 & 011110 & $\begin{array}{lllllllllllllll}0 & 0 & 0 & 1 & 0 & 0\end{array}$ & $\begin{array}{lllll}1 & 0 & 1 & 1 & 1\end{array}$ & $0 \quad 11110$ \\
\hline 5 & $\begin{array}{llllllllll}0 & 1 & 0 & 1 & 0\end{array}$ & $\begin{array}{lllll}0 & 0 & 1 & 1 & 0\end{array}$ & 111110 & $\begin{array}{lllll}0 & 1 & 0 & 1 & 0\end{array}$ & $\begin{array}{llllll}0 & 0 & 1 & 1 & 0\end{array}$ \\
\hline 6 & $\begin{array}{lllll}0 & 0 & 0 & 0 & 0\end{array}$ & 100011 & $\begin{array}{llllll}1 & 0 & 1 & 0 & 1 & 1\end{array}$ & $\begin{array}{lllll}0 & 0 & 0 & 0 & 0\end{array}$ & 100011 \\
\hline 7 & $\begin{array}{llllll}0 & 1 & 1 & 1 & 1\end{array}$ & 10110 & $\begin{array}{lllllllll}0 & 0 & 1 & 0 & 1 & 0\end{array}$ & $\begin{array}{lllllll}0 & 1 & 1 & 1 & 1\end{array}$ & 100110 \\
\hline 8 & $\begin{array}{llllll}0 & 0 & 0 & 0 & 1\end{array}$ & 11011 & $\begin{array}{llllllll}0 & 1 & 0 & 0 & 0 & 1\end{array}$ & $\begin{array}{llllll}0 & 0 & 0 & 0 & 1\end{array}$ & 11011 \\
\hline
\end{tabular}

In (1), (.)* denotes complex conjugation. The Alamouti matrix is unitary, i.e., $\mathbf{B}^{H} \mathbf{B}=c \mathbf{I}_{2}$, where $\mathbf{B}^{H}$ is the Hermitian conjugate of $\mathbf{B}, c$ denotes a complex-valued constant, and $\mathbf{I}_{2}$ the $(2 \times 2)$ identity matrix.

Fig. 1(b) shows the transmitter structure of the GSM/EDGE system enhanced by the Alamouti scheme. The data symbols $x(k)$ provided by the modulation and coding scheme are space-time processed according to (1) and mapped on GSM/EDGE bursts. The bursts intended for the first and the second transmit antenna are equipped with training sequences TS1 and TS2, respectively. In contrast to the delay-diversity scheme, two different TSs are mandatory. Finally, pulse shaping is done and the bursts are transmitted over the two antennas.

As in the case of the delay-diversity scheme, the single transmit antenna case is included in the enhanced structure as the special case, when the second transmit antenna is switched off. In turn, the data rate of the $(1 \times 1)$ system is retained.

\section{Receiver Structure}

Fig. 1(c) shows the general receiver structure for the case of a single receive antenna $\left(n_{R}=1\right)$ applying both for the $(1 \times 1)$ system and for the enhanced systems. The received signal $r(t)$ is first filtered (Rx-filter) and then sampled at time instants $k T$ yielding received symbols $y^{\prime}(k)$. The channel estimator provides estimates of the coefficients of the equivalent discrete-time channel model, here referred to as channel coefficients. They are obtained on the basis of the derotated received symbols $y(k)$ exploiting knowledge of the TS(s) used. Eventually, equalization and detection is performed on the basis of the channel estimates. The employed Max-Log-MAP algorithm is a soft-output detector, which approximates the log-likelihood ratios (LLRs) $L(x(k))$ of the data symbols $x(k)$.

\section{Data Detection in the Case of the Alamouti Scheme}

In order to detect the transmitted data symbols, the equalizer-detector employed in the receiver requires knowledge about the channel coefficients. In a GSM/EDGE receiver, channel estimation (CE) is typically performed by means of the correlation method [23]. In this method, the channel estimates are obtained by correlating the employed TS(s) with the corresponding received symbols.

If a single TS is employed, i.e., in the $(1 \times 1)$ system and in the system enhanced by delay diversity, solely the auto-correlation properties of the TS used are crucial for the quality of the resulting estimates (if co-channel interference is neglected). The eight GSM sequences [see Table I(A)] are characterized by perfect auto-correlation properties for shifts $-5 \leq \xi \leq+5$ and, thus, grant-with respect to the average estimation error-optimal channel estimates $\hat{\mathbf{h}}=\left[\hat{h}^{(0)}, \hat{h}^{(1)}, \ldots, \hat{h}^{(L)}\right]$ for channels with a memory length $L \leq 5$.

In the case of the Alamouti scheme, where TS1 and TS2 are required [cf. Fig. 1(b)], not only the auto-correlation properties of both sequences determine the quality of the channel estimates, but their cross-correlation properties as well. The correlation of TS1 and TS2 with the received midamble symbols yields channel coefficient estimates $\hat{\mathbf{h}}_{\mathbf{1}}$ and $\hat{\mathbf{h}}_{\mathbf{2}}$, respectively, which describe the transmission path corresponding to the first and to the second transmit antenna.

TheETSI/3GPP specifications [11] donot comprise any partner sequences with respect to the eight GSM sequences at all. Pairs of GSM sequences are in fact characterized by comparably poor cross-correlation properties. Moreover, the existing eight TSs are already invariably required in the single transmit antennacase, due to the cellular structure of a GSM radio network. It, therefore, pays off to define adequate TS pairs for the Alamouti case.

\section{A. Definition of Appropriate TS Pairs}

In Table I(B), optimized partner sequences with respect to the eight GSM TSs are listed. These sequences have perfect auto-correlation properties, the same length as the GSM sequences (26 symbols), and the same cyclic structure "A B C A B." Among all sequences with these properties, the ones stated in Table $\mathrm{I}(\mathrm{B})$ are optimal in the sense of minimizing the mean squared errors 
$E\left\{\left\|\mathbf{h}_{\mathbf{1}}-\hat{\mathbf{h}}_{\mathbf{1}}\right\|^{2}\right\}$ and $E\left\{\left\|\mathbf{h}_{\mathbf{2}}-\hat{\mathbf{h}}_{\mathbf{2}}\right\|^{2}\right\}$, provided that $\hat{\mathbf{h}}_{\mathbf{1}}$ and $\hat{\mathbf{h}}_{\mathbf{2}}$ are obtained by means of the correlation method and a TU wireless channel $(L=3)$ is given. In the case of a channel memory length $L \leq 2$, even optimal $\mathrm{CE}$ is accomplished; i.e., the resulting sequence pairs are characterized by perfect cross-correlation properties for shifts $-2 \leq \xi \leq+2$.

Simulation results indicate that-with regard to the resulting bit-error performance of the overall system-the proposed sequence pairs significantly outperform any pair of GSM sequences (refer to Section V-B). As a matter of fact, the performance accomplished on a noiseless TU channel is very close to the case when all channel coefficients are perfectly known at the receiver.

An alternative approach to the one pursued here can be adopted from [24]. Given a certain GSM TS1, the idea is to construct a partner sequence by applying to TS1 a cyclic shift of $K>L$ symbols. Provided that, e.g., $K=L+1$, altogether $2(L+1)$ channel coefficients are estimated by correlating TS1 with the received midamble symbols. Then, $\hat{\mathbf{h}}_{\mathbf{1}}$ and $\hat{\mathbf{h}}_{\mathbf{2}}$ are given by the first $L+1$ and the second $L+1$ estimates, respectively. However, optimal CE is in turn only accomplished for a channel memory length $L \leq 2$ since the auto-correlation properties of the GSM sequences enable optimal estimation of altogether six channel coefficients only. In the TU case, for example, one will obtain virtually no information about the channel coefficients $h_{2}^{(2)}$ and $h_{2}^{(3)}$ of the second transmission path, which leads to a serious performance loss.

\section{B. Generalization of the Max-Log-MAP Algorithm}

For the GSM/EDGE system enhanced by delay diversity virtually the same equalization and detection algorithm as in the $(1 \times 1)$ system can be employed, provided that the algorithm is capable of handling the increased memory length (see Section IV-A1). Within the scope of this work, we concentrate on the Max-Log-MAP algorithm [12]. In the case of the Alamouti scheme, the conventional Max-Log-MAP algorithm has to be modified in a way that - corresponding to the transmitter structure-the received symbols are processed as pairs.

The Max-Log-MAP algorithm is a trellis-based algorithm and approximates the LLRs $L(x(k))$ of the transmitted data symbols $x(k)$. LLRs provide "soft" reliability information about each individual symbol decision, which may, in a coded system, be utilized by an outer channel decoder. As opposed to the well-known Viterbi algorithm [21, Ch. 10.1], which performs maximum-likelihood sequence estimation, the Max-Log-MAP algorithm is based on a symbol-by-symbol MAP criterion. For both algorithms, however, the same trellis diagram and the same branch metric computation can be used. For reasons of brevity, we will not recapitulate the definition of the Max-Log-MAP algorithm with its computation of the $L(x(k))$ here, but rather outline the main differences between the conventional algorithm and the generalized Max-Log-MAP algorithm required in the Alamouti case. Since these differences mainly concern the resulting trellis structure and the metric computation, the concept presented in the following applies in principle as well for other trellis-based detection algorithms, such as the Viterbi or the BCJR algorithm ${ }^{1}$ [25].

\footnotetext{
${ }^{1}$ The BCJR algorithm is named after its inventors Bahl, Cocke, Jelinek, and Raviv and performs symbol-by-symbol MAP detection.
}

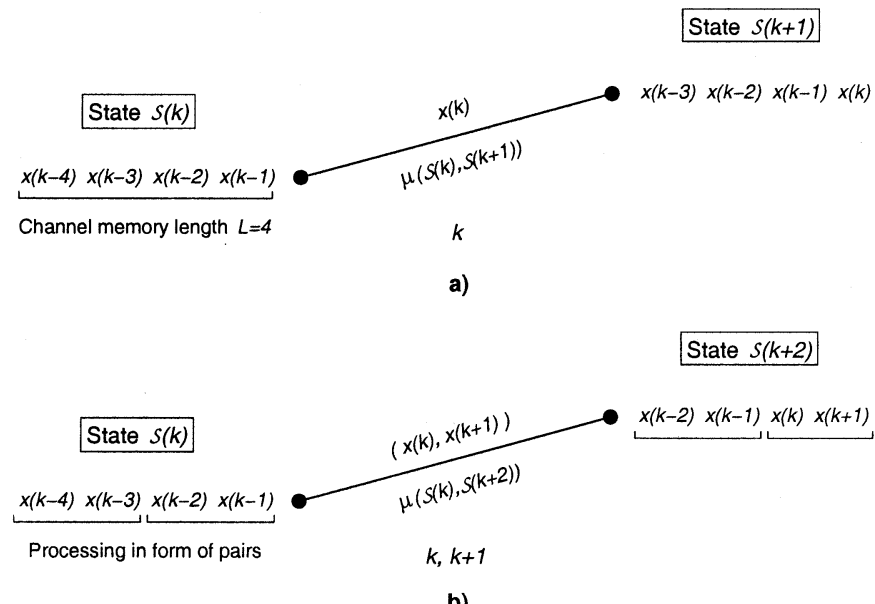

b)

Fig. 2. State transition (a) within the conventional trellis (channel memory length $L=4)$ and (b) within the Alamouti-trellis $(L=4)$.

In Fig. 2, a comparison is drawn between the conventional trellis and the trellis resulting for the Alamouti scheme with regard to trellis states and transitions (channel memory length $L=4$ in both cases). In the first case, a trellis segment complies with a single data symbol $x(k)$. Presuming a single receive antenna $\left(n_{R}=1\right)$, the Euclidean branch metric $\mu(\mathcal{S}(k), \mathcal{S}(k+1))$ for a transition $\mathcal{S}(k) \rightarrow \mathcal{S}(k+1)$ is given by $|y(k)-\tilde{y}(k)|^{2}$, where $y(k)$ denotes the current received symbol and $\tilde{y}(k)$ a corresponding hypothesis. $\tilde{y}(k)$ is a replica of $y(k)$, determined by the state $\mathcal{S}(k)$, the symbol hypothesis $\tilde{x}(k)$ associated with the transition regarded, and the (estimated) channel coefficients at time index $k$. The number of possible trellis states $\mathcal{S}(k)$ is $M^{L}$, where $M$ is the cardinality of the symbol alphabet.

In the Alamouti scheme a single trellis segment spans two consecutive time indexes $k$ and $k+1$. A transition from a state $\mathcal{S}(k)$ to a subsequent state $\mathcal{S}(k+2)$, therefore, complies with a pair $(x(k), x(k+1))$ of data symbols. As in the conventional trellis, the number of possible trellis states $\mathcal{S}(k)$ is given by $M^{L}$, where $L$ is even. This means that both detection algorithms are of same complexity. ${ }^{2}$ The Euclidean branch metric $\mu(\mathcal{S}(k), \mathcal{S}(k+2))$ for a transition $\mathcal{S}(k) \rightarrow \mathcal{S}(k+2)$ is calculated according to

$\mu(\mathcal{S}(k), \mathcal{S}(k+2))=|y(k)-\widetilde{y}(k)|^{2}+|y(k+1)-\widetilde{y}(k+1)|^{2}$.

Let the vectors of the channel coefficient estimates be given by $\hat{\mathbf{h}}_{\mathbf{1}}=\left[\hat{h}_{1}^{(0)}, \ldots, \hat{h}_{1}^{(L)}\right]$ and $\hat{\mathbf{h}}_{\mathbf{2}}=\left[\hat{h}_{2}^{(0)}, \ldots, \hat{h}_{2}^{(L)}\right]$ (first and second transmit antenna, respectively). The hypotheses $\tilde{y}(k)$ and $\tilde{y}(k+1)$ are then calculated as follows:

$$
\begin{gathered}
\tilde{y}(k)=\sum_{\kappa=0}^{L}\left[\hat{h}_{1}^{(\kappa)} \tilde{x}(k-\kappa)-\left.\hat{h}_{2}^{(\kappa)} \tilde{x}^{*}(k-(\kappa-1))\right|_{\kappa \text { even }}\right. \\
\left.+\left.\hat{h}_{2}^{(\kappa)} \tilde{x}^{*}(k-(\kappa+1))\right|_{\kappa \text { odd }}\right]
\end{gathered}
$$

${ }^{2}$ If $L$ is odd, $M^{L+1}$ states are required leading to a higher complexity compared with the conventional trellis (a statement for general STBC of Rate 1 is given in [27, Ch. 7.3.4.4]). 


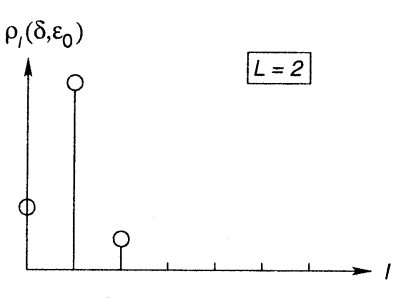

a)

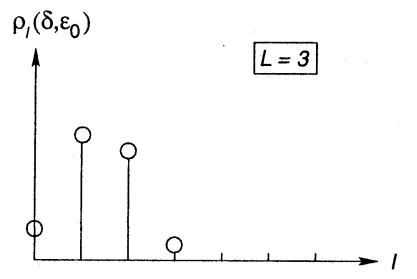

b)

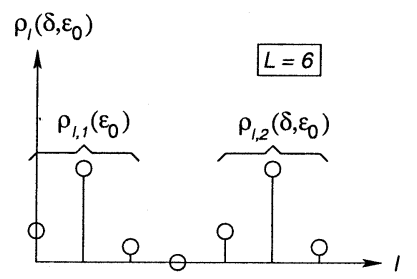

c)

Fig. 3. Mean power $\rho_{l}(\delta, \epsilon)$ of the channel coefficients $\left(n_{T}=2\right.$ transmit antennas, sampling phase $\left.\epsilon=\epsilon_{0}\right)$. (a) Delay $\delta=0$. (b) Delay $\delta=T$. (c) Delay $\delta=4 T$.

and

$$
\begin{aligned}
\tilde{y}(k+1)=\sum_{\kappa=0}^{L}[ & \hat{h}_{1}^{(\kappa)} \tilde{x}((k+1)-\kappa) \\
& +\left.\hat{h}_{2}^{(\kappa)} \tilde{x}^{*}((k+1)-(\kappa+1))\right|_{\kappa \text { even }} \\
& \left.\quad-\left.\hat{h}_{2}^{(\kappa)} \tilde{x}^{*}((k+1)-(\kappa-1))\right|_{\kappa \text { odd }}\right] .
\end{aligned}
$$

The symbol hypotheses $\tilde{x}(k-L), \ldots, \tilde{x}(k-1)$ are determined by the state $\mathcal{S}(k)$ and the hypotheses $\tilde{x}(k), \tilde{x}(k+1)$ by the transition regarded.

In the case of multiple receive antennas, a partial branch metric $\mu_{j}(\mathcal{S}(k), \mathcal{S}(k+2))$ is calculated for each individual receive antenna $1 \leq j \leq n_{R}$, which is done corresponding to (2) on the basis of the received symbols $y_{j}(k), y_{j}(k+1)$ and the corresponding hypotheses $\tilde{y}_{j}(k)$ and $\tilde{y}_{j}(k+1)$. The $\tilde{y}_{j}(k)$ and $\tilde{y}_{j}(k+1)$ are obtained according to (3), where each receive antenna $j$ is associated with dedicated vectors $\hat{\mathbf{h}}_{\mathbf{1} \mathbf{j}}, \hat{\mathbf{h}}_{\mathbf{2} \mathbf{j}}$ of channel coefficient estimates. The overall branch metric $\mu$ is obtained by a sum over the partial metrics $\mu_{j}$

$$
\mu(\mathcal{S}(k), \mathcal{S}(k+2))=\sum_{j=1}^{n_{R}} \mu_{j}(\mathcal{S}(k), \mathcal{S}(k+2))
$$

\section{ANALYTICAL Results}

\section{A. RRB for the Delay-Diversity Scheme}

The RRB [21, Ch. 14.5] is a lower bound on the bit-error probability of a slowly time-varying frequency-selective channel, where perfect knowledge of the channel coefficients at the receiver is assumed. Performance loss due to intersymbol interference (ISI) is not taken into account [26]. All channel coefficients are assumed to fade independently.

In order to compute the RRB, the mean power $\rho_{l} \doteq E\left\{\left|h^{(l)}(k)\right|^{2}\right\}, 0 \leq l \leq L$, of the channel coefficients $\left[h^{(0)}(k), h^{(1)}(k), \ldots, h^{(L)}(k)\right]$ is required $(E\{\}$. denotes the expected value and $k$ the time index). In the case of the delay-diversity scheme with $n_{T}=2$ transmit antennas, $\rho_{l}$ is a function of the delay $\delta$ applied to the signal at the second transmit antenna. In the following, an analytical expression is derived for $\rho_{l}$, which will later be utilized in order to find an optimal delay $\delta$ minimizing the RRB.

1) Mean Power of the Channel Coefficients: Since in the delay-diversity scheme the same signal is transmitted over each antenna, the transmission paths from either transmit antenna $1 \leq i \leq n_{T}$ to a certain receive antenna $1 \leq j \leq n_{R}$ can be combined in a joint channel model [27, Ch. 7.3.1], where the delays $\delta_{i}$ applied at the different transmit antennas need to be taken into account. In the case of $n_{T}=2$ and $n_{R}=1$, the overall channel model can be described by a vector $\mathbf{h}(k, \delta)=\left[h^{(0)}(k, \delta), h^{(1)}(k, \delta), \ldots, h^{(L)}(k, \delta)\right]$ of channel coefficients. Therefore, the received symbols are given by

$$
y(k)=\sum_{l=0}^{L} h^{(l)}(k, \delta) x(k-l)+n(k)
$$

where $x(k)$ denotes the $k$ th data symbol, $n(k)$ denotes an additive white Gaussian noise (AWGN) sample, and $h^{(l)}(k, \delta)=$ $h_{1}^{(l)}(k)+h_{2}^{(l-m)}(k)$ for the special case that $\delta=m T$ with $m$ being an integer number.

The derivation of $\rho_{l}$ is done corresponding to the way described in [26, Appendix II]. One obtains

$$
\begin{aligned}
\rho_{l}(\delta, \epsilon) & =\int_{0}^{\tau_{\max }} p(\tau)\left(\left|g_{\operatorname{TxRx}}(l T-\tau-\epsilon)\right|^{2}\right. \\
& \left.\quad+\left|g_{\operatorname{TxRx}}(l T-\delta-\tau-\epsilon)\right|^{2}\right) d \tau \\
\doteq & \rho_{l, 1}(\epsilon)+\rho_{l, 2}(\delta, \epsilon), \quad 0 \leq l \leq L
\end{aligned}
$$

where $g_{\operatorname{TxRx}}(\xi) \doteq g_{T x}(\xi) * g_{R x}(\xi)$ denotes the overall impulse response of transmitter and receiver comprising a pulse shaping filter $g_{T x}(\xi)$ and a receiver filter $g_{R x}(\xi)$ (the asterisk means convolution). The power density function (pdf) $p(\tau)$, $0 \leq \tau \leq \tau_{\max }$, is proportional to the delay power density profile. An example for the delay power density profile is the GSM 05.05 propagation profiles [11]. The pdf $p(\tau)$ is assumed to apply for both transmission paths. Corresponding to [26], an additional sampling phase $\epsilon \in \mathbb{R}$ is taken into account, where $0 \leq \epsilon<T$. The values $\rho_{l}(\delta, \epsilon)$ are normalized such that for any $\delta, \epsilon$

$$
\sum_{l=0}^{L} \rho_{l}(\delta, \epsilon)=1 .
$$

Fig. 3 illustrates $\rho_{l}$ resulting for different delays $\delta$ and a fixed sampling phase $\epsilon_{0}$. Note that the channel memory length $L$ resulting for the joint channel model is actually a function of $\delta$. The diversity gain accomplished by means of delay diversity $(\delta>0)$ is, therefore, due to an increased degree of frequency selectivity.

In the following, a trellis-based equalizer-detector of length $L_{\mathrm{eq}}$ is assumed to be employed in the receiver; i.e., in the branch metric computations, the first $\left(L_{\mathrm{eq}}+1\right)$ channel coefficients of the vector $\mathbf{h}(k, \delta)$ are taken into account $\left(L_{\mathrm{eq}} \leq L\right)$. 


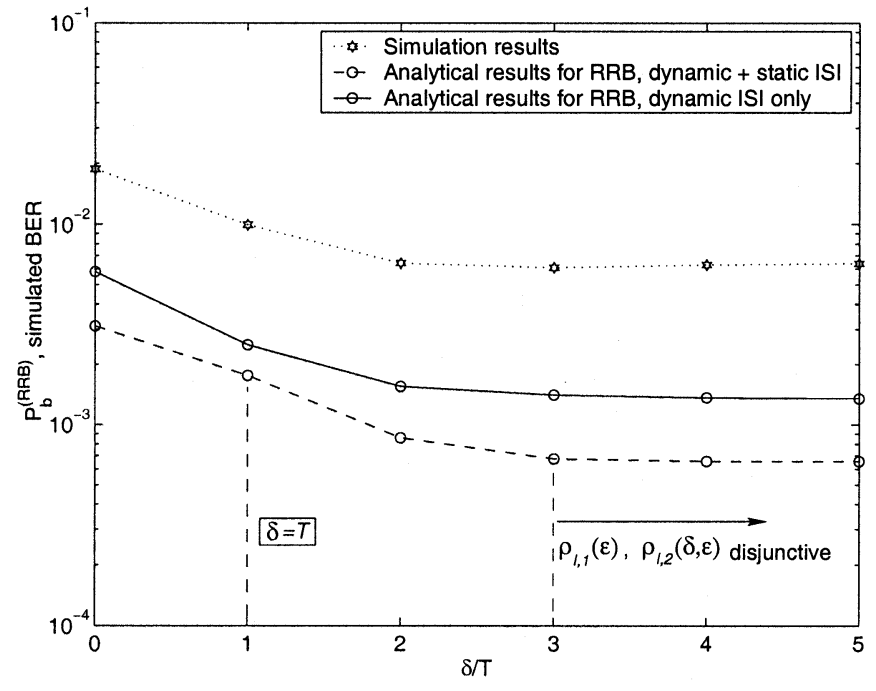

Fig. 4. RRB as a function of $\delta$, equalizer-detector length $L_{\mathrm{eq}}=L, \mathrm{TU}$ profile, $E_{s} / N_{0}=10 \mathrm{~dB}$, sampling phase $\epsilon=0$.

2) RRB Resulting for an Optimal Trellis-Based Equalizer-Detector: In the case of a binary modulation scheme and an equalizer-detector of length $L_{\mathrm{eq}}=L$, the RRB is given by

$$
\begin{aligned}
& P_{b}^{(\mathrm{RRB})}=\frac{1}{2} \sum_{\lambda=0}^{L}\left(\prod_{\substack{\nu=0 \\
\rho_{\nu} \neq \rho_{\lambda}}}^{L} \frac{\rho_{\lambda}(\delta, \epsilon)}{\rho_{\lambda}(\delta, \epsilon)-\rho_{\nu}(\delta, \epsilon)}\right) \\
& \cdot\left(1-\frac{1}{\sqrt{1+\frac{N_{0}}{E_{s}} \frac{1}{\rho_{\lambda}(\delta, \epsilon)}}}\right)
\end{aligned}
$$

where $E_{s}$ denotes the mean energy per data symbol and $N_{0}$ the single-sided noise power density. For the derivation of (8), refer to [21, Ch. 14.5]. Note that the RRB is a function of $\delta, \epsilon$, and the signal-to-noise ratio (SNR) $E_{s} / N_{0}$.

In Fig. 4, the RRB is plotted as a function of $\delta$ (dashed line) resulting for the GSM 05.05 TU propagation profile, GMSK modulation, a sampling phase $\epsilon=0$, and an SNR of $10 \mathrm{~dB}$. Throughout the paper, a nonadaptive root-raised-cosine receiver filter is considered (roll-off factor $r=0.5,3-\mathrm{dB}$ bandwidth $f_{3 \mathrm{~dB}}=180 \mathrm{kHz}$ ). Fig. 4 shows that within the interval $0 \leq$ $\delta \leq 3 T$ the RRB decreases with growing delay $\delta$, i.e., with an increased degree of frequency selectivity. Note that the choice $\delta=T$ typically made in a delay-diversity scheme with two transmit antennas is not optimal here. For $\delta=3 T$, the two terms $\rho_{l, 1}(\epsilon)$ and $\rho_{l, 2}(\delta, \epsilon)$ in $\rho_{l}$ according to (6) are virtually disjunctive in the time domain [cf. Fig. 3(c)], which leads to the maximum possible diversity gain. Therefore, delays $\delta>$ $3 T$ solely increase the resulting channel memory length $L$ but do not accomplish further diversity gain. Accordingly, the RRB does not decrease further for $\delta>3 T$. Corresponding simulation results also included in Fig. 4 (dotted line) are in accordance with the general shape of the RRB curve.

As Fig. 4 illustrates, the conventional RRB leads to bit-error probabilities that are significantly smaller than the simulated bit-error rates (BERs). This is due to the fact that the RRB presumes independent fading of the individual channel coefficients corresponding to the $\rho_{l}$. However, the channel coeffi- cients are characterized by both dynamic ISI, which is due to the frequency-selective fading channel, and static ISI, which is due to the overall impulse response of transmitter and receiver $g_{\mathrm{TxRx}}(\xi)$, i.e., the assumption of independent fading is not valid. This means the RRB overestimates the degree of diversity utilized and, therefore, yields bit-error probabilities that are too optimistic.

For the purpose of analysis, the static ISI can be eliminated from $\rho_{l}$ by means of a linear zero-forcing (ZF) equalizer. The equalizer can be characterized by a finite-impulse-response filter structure and its coefficients $e_{\mathrm{ZF}}^{(\epsilon)}(l)$ are matched to the samples $g_{\mathrm{TxRx}}(l T-\epsilon) \doteq g^{(\epsilon)}(l)$ of $g_{\mathrm{TxRx}}(\xi)$. The convolution of the original channel coefficients and the ZF equalizer yields a set of modified channel coefficients $\tilde{h}^{(l)}(k, \delta)(0 \leq l \leq \tilde{L})$, which are solely characterized by the dynamic ISI. The mean power $\tilde{\rho}_{l}$ of the modified channel coefficients is given by

$$
\begin{aligned}
& \tilde{\rho}_{l}(\delta, \epsilon)=\int_{0}^{\tau_{\max }} p(\tau)\left(\left|g_{\operatorname{TxRx}}(l T-\tau-\epsilon) * e_{\mathrm{ZF}}^{(\epsilon)}(l)\right|^{2}\right. \\
&\left.+\left|g_{\operatorname{TxRx}}(l T-\delta-\tau-\epsilon) * e_{\mathrm{ZF}}^{(\epsilon)}(l)\right|^{2}\right) d \tau \\
& \doteq \tilde{\rho}_{l, 1}(\epsilon)+\tilde{\rho}_{l, 2}(\delta, \epsilon),
\end{aligned}
$$

where $0 \leq l \leq \tilde{L}$. The derivation of (9) is given in Appendix A. In turn, the $\tilde{\rho}_{l}$ are normalized such that their sum, according to (7), is one. Note again that the ZF equalizer is only employed for the purpose of analysis. Particularly, noise enhancement and noise coloring do not affect the analysis at all.

The RRB curve computed on the basis of $\tilde{\rho}_{l}$ is shown in Fig. 4 as well (solid line). The general shape of the curve corresponds to that obtained for $\rho_{l}$. However, due to the fact that the static ISI has been removed by means of the ZF equalizer, the bit-error probabilities of the new RRB curve are less optimistic and, therefore, closer to the simulation results. Yet it is important to note that the channel coefficients $\tilde{h}^{(l)}(k, \delta)$ still do not fade independently, as filtering with the ZF equalizer leads to residual correlation between the $\tilde{h}^{(l)}(k, \delta)$.

The influence of the SNR $\left(E_{s} / N_{0}\right)$ on the improved RRB is such that with growing SNR the RRB tends to diminish. For example, for large $\delta$ and an SNR of 6 and $16 \mathrm{~dB}$, bit-error probabilities of $1.3 \cdot 10^{-2}$ and $7 \cdot 10^{-6}$ result, respectively. In the first case, the bit-error probabilities span approximately $1 / 3$ decade for $\delta=0, \ldots, 5 T$ and in the latter case 1.5 decades.

3) RRB Resulting for a Truncated Trellis-Based Equalizer-Detector: In the following, we focus on the improved RRB curve. The case of a trellis-based equalizer-detector of length $L_{\mathrm{eq}}<\tilde{L}$ corresponds to a modified channel model, which is characterized by only $\left(L_{\text {eq }}+1\right)$ channel coefficients and a transformed SNR denoted as $\left(E_{s} / N_{0}\right)^{\prime}$

$$
\begin{aligned}
P_{b}^{(\mathrm{RRB})}=\frac{1}{2} \sum_{\lambda=0}^{L_{\mathrm{eq}}}\left(\prod_{\substack{\nu=0 \\
\tilde{\rho}_{\nu} \neq \tilde{\rho}_{\lambda}}}^{L_{\mathrm{eq}}} \frac{\tilde{\rho}_{\lambda}(\delta, \epsilon)}{\tilde{\rho}_{\lambda}(\delta, \epsilon)-\tilde{\rho}_{\nu}(\delta, \epsilon)}\right) \\
\cdot\left(\begin{array}{l}
\left.1-\frac{1}{\sqrt{1+\left(\frac{N_{0}}{E_{s}}\right)^{\prime} \frac{1}{\tilde{\rho}_{\lambda}(\delta, \epsilon)}}}\right)
\end{array}\right.
\end{aligned}
$$




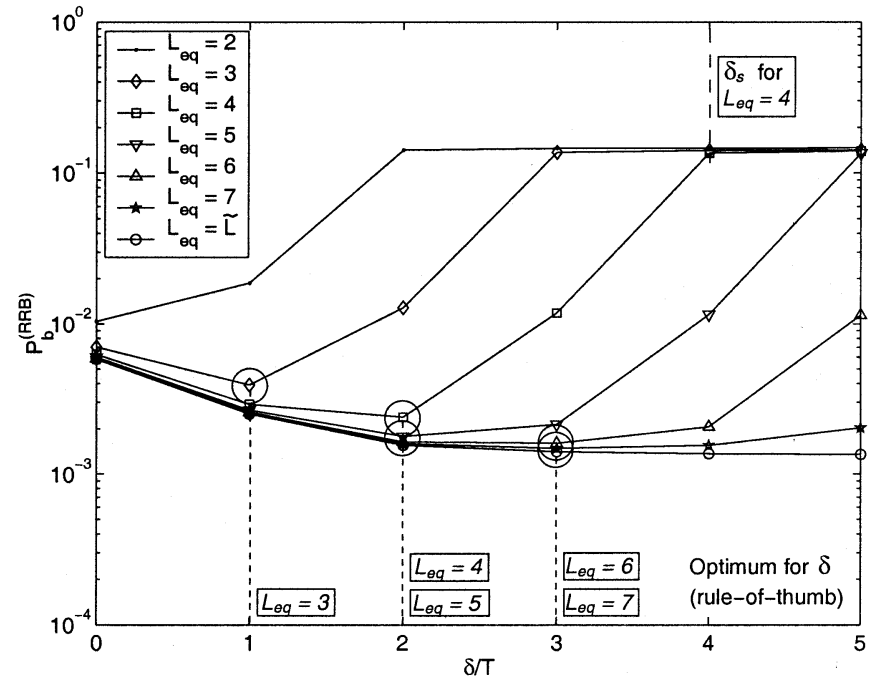

Fig. 5. RRB as a function of $\delta$, different equalizer-detector lengths $L_{\text {eq }}$, TU profile, $E_{s} / N_{0}=10 \mathrm{~dB}$, sampling phase $\epsilon=0$ (analytical results).

where $\left(N_{0} / E_{s}\right)^{\prime}$ comprises the mean energy of the residual ISI term, which results from the fact that the channel coefficients $\left[\tilde{h}^{\left(L_{\text {eq }}+1\right)}(k, \delta), \ldots, \tilde{h}^{(\tilde{L})}(k, \delta)\right]$ are neglected in the modified channel model. One obtains

$$
\left(\frac{N_{0}}{E_{s}}\right)^{\prime}=\frac{N_{0}}{E_{s}}+\sum_{l=L_{\mathrm{eq}}+1}^{\tilde{L}} \tilde{\rho}_{l}(\delta, \epsilon)
$$

For the derivation of (11), see Appendix B.

Fig. 5 shows the RRB as a function of $\delta$ resulting for different equalizer-detector lengths $L_{\mathrm{eq}}$ (GSM 05.05 TU profile, $E_{s} / N_{0}=10 \mathrm{~dB}$, sampling phase $\epsilon=0$ ). With growing $\delta$, the RRB curve for a given $L_{\text {eq }}$ complies with the ideal curve $\left(L_{\mathrm{eq}}=\tilde{L}\right)$, as long as the equalizer-detector metric spans the predominant fraction of the sum in (7), i.e., $\left(\tilde{L}-L_{\text {eq }}\right)$ is sufficiently small. Greater values of $\delta$ lead to degradation of the RRB due to residual ISI. At a certain delay $\delta_{s}$, the equalizer-detector metric spans solely that fraction of (7) which corresponds to the term $\tilde{\rho}_{l, 1}(\epsilon)$ in $\tilde{\rho}_{l}$ [cf. (9)]. Therefore, delays $\delta>\delta_{s}$ do not lead to further degradation of the RRB.

4) Optimization of the Delay Parameter: Given an optimal equalizer-detector of length $L_{\text {eq }}=\tilde{L}$, the delay $\delta$ applied to the signal at the second transmit antenna should be $\delta \geq 3 T$ in the TU case, so as to minimize the RRB (cf. Fig. 4). In the case of an equalizer-detector of a fixed length $L_{\text {eq }}$, a rule-of-thumb can be derived from Fig. 5 concerning the optimal choice of $\delta$ (sampling phase $\epsilon=0, L_{\text {eq }} \geq 3$ )

$$
\delta_{\text {opt }} \approx\left\lfloor L_{\text {eq }} / 2\right\rfloor \cdot T
$$

where $\lfloor x\rfloor$ is the greatest integer value $i$ satisfying $i \leq x$. The values resulting for $\delta_{\text {opt }}$, given different equalizer-detector lengths, are included in Fig. 5. Since (12) has been derived on the basis of the modified channel model comprising the ZF equalizer, it is important to note that it applies as well for the original channel model, which-from a practical point of view-is the more relevant one.

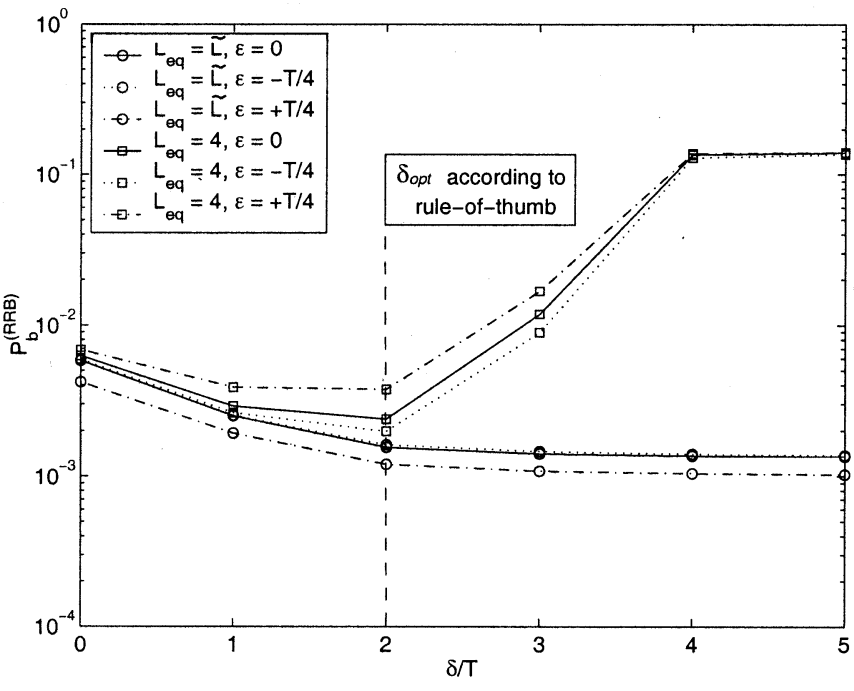

Fig. 6. Influence of the sampling phase $\epsilon$ on the RRB, TU profile, $E_{s} / N_{0}=$ $10 \mathrm{~dB}$ (analytical results).

Fig. 6 illustrates the influence of the sampling phase $\epsilon$ on the RRB, both for an optimal and a truncated trellis-based equalizer-detector $\left(L_{\mathrm{eq}}=4\right)$. In both cases, variations of $\epsilon$ between $\pm T / 4$ lead to a slightly different shape of the curve. Nevertheless, the rule-of-thumb (12) still applies.

\section{B. Orthogonal Properties of the Alamouti Scheme}

The Alamouti scheme has been designed for quasi-static flatfading channels. In the following, it is shown that the orthogonal properties of the Alamouti scheme are lost in the case of nonperfect knowledge of the channel coefficients at the receiver as well as in the presence of fast-fading or frequency-selective fading. This orthogonality loss will cause a performance degradation. The results obtained in the following hold, in principle, for an arbitrary STBC.

First of all, consider a quasi-static flat-fading channel assuming $n_{R}=1$ receive antenna. In this case, the two transmission paths from either transmit antenna to the receive antenna can be modeled by means of two complex-valued channel coefficients $h_{1}$ and $h_{2}$ (channel memory length $L=0$ ), which are constant over the duration of a certain block of data (e.g., over an entire GSM burst). Taking into account the symbol mapping according to matrix $\mathbf{B}$, the received symbols $y(k)$ and $y(k+1)$ at the time instants $k$ and $k+1$ are given by the following matrix equation:

$\underbrace{\left[\begin{array}{c}y(k) \\ y^{*}(k+1)\end{array}\right]}_{\mathbf{y}(k)}=\underbrace{\left[\begin{array}{rr}h_{1} & -h_{2} \\ h_{2}^{*} & h_{1}^{*}\end{array}\right]}_{\mathbf{H}} \underbrace{\left[\begin{array}{c}x(k) \\ x^{*}(k+1)\end{array}\right]}_{\mathbf{x}(k)}+\underbrace{\left[\begin{array}{c}n(k) \\ n^{*}(k+1)\end{array}\right]}_{\mathbf{n}(k)}$

where $n(k)$ and $n(k+1)$ denote samples of AWGN occurring at time index $k$ and $k+1$, respectively. $\mathbf{H}$ is unitary, which is due to the unitarity of the Alamouti matrix.

The detection of the symbols $x(k)$ and $x(k+1)$ can simply be performed by means of the matrix-vector multiplication $\mathbf{H}^{H} \mathbf{y}(k)$. In this context-assuming perfect knowledge of $h_{1}$ and $h_{2}$ at the receiver-the unitarity of $\mathbf{H}$ leads to a decoupling 
of $x(k)$ and $x(k+1)$ in terms of independent estimates $\hat{x}(k)$ and $\hat{x}(k+1)$ (see, e.g., [27, Ch. 7.3.2])

$$
\hat{\mathbf{x}}(k)=\mathbf{H}^{H} \mathbf{y}(k)=\mathbf{H}^{H} \mathbf{H} \mathbf{x}(k)+\mathbf{H}^{H} \mathbf{n}(k)
$$

where

and

$$
\hat{\mathbf{x}}(k)=\left[\begin{array}{c}
\hat{x}(k) \\
\hat{x}^{*}(k+1)
\end{array}\right]
$$

$$
\mathbf{H}^{H} \mathbf{H}=\left(\left|h_{1}\right|^{2}+\left|h_{2}\right|^{2}\right) \mathbf{I}_{2} .
$$

Computing $\hat{\mathbf{x}}(k)$ according to (14) is equivalent to minimizing the metric (2) for $L=0$. Note that due to the diagonal structure of $\mathbf{H}^{H} \mathbf{H}$, the desired symbols are always combined in a constructive way because they are multiplied by a sum of absolute terms. The noise, however, is combined incoherently (matrix $\left.\mathbf{H}^{H}\right)$, which leads to a diversity gain over a $(1 \times 1)$ system.

1) Orthogonality Loss Due to Nonperfect CE: Let $\hat{h}_{1}$ and $\hat{h}_{2}$ denote noisy estimates of the channel coefficients $h_{1}$ and $h_{2}$, where $\hat{h}_{1} \doteq h_{1}+\varepsilon_{1}$ and $\hat{h}_{2} \doteq h_{2}+\varepsilon_{2}$. The detection of the symbols $x(k)$ and $x(k+1)$ will then be performed using

$$
\hat{\mathbf{H}}=\left[\begin{array}{rr}
\hat{h}_{1} & -\hat{h}_{2} \\
\hat{h}_{2}^{*} & \hat{h}_{1}^{*}
\end{array}\right]
$$

i.e., (14) becomes

$$
\hat{\mathbf{x}}(k)=\hat{\mathbf{H}}^{H} \mathbf{H} \mathbf{x}(k)+\hat{\mathbf{H}}^{H} \mathbf{n}(k) .
$$

Contrary to (14), $\hat{\mathbf{H}}^{H} \mathbf{H}$ does not yield a diagonal matrix

$$
\hat{\mathbf{H}}^{H} \mathbf{H}=\left[\begin{array}{rr}
\psi & \varsigma^{*} \\
-\varsigma & \psi^{*}
\end{array}\right]
$$

where

$$
\psi=\left|h_{1}\right|^{2}+\left|h_{2}\right|^{2}+\varepsilon_{1}^{*} h_{1}+\varepsilon_{2} h_{2}^{*}
$$

and

$$
\varsigma=\varepsilon_{2}^{*} h_{1}-\varepsilon_{1} h_{2}^{*} .
$$

Despite this loss of orthogonality, the desired symbols $x(k)$ and $x(k+1)$ are still combined in a constructive way. They are, however, weighted with different factors, as the diagonal elements of $\hat{\mathbf{H}}^{H} \mathbf{H}$ are not equal. The secondary diagonal elements $-\varsigma$ and $\varsigma^{*}$ introduce an additional error term to the estimates $\hat{x}(k)$ and $\hat{x}(k+1)$, which is solely due to the loss of orthogonality and does, therefore, not occur in a $(1 \times 1)$ system. Note that for $\varepsilon_{1}, \varepsilon_{2} \rightarrow 0$, (16) reduces to (14).

2) Orthogonality Loss Due to Fast Fading: In case of fast fading, the channel coefficients $h_{1}$ and $h_{2}$ may vary over the duration of a symbol pair $(x(k), x(k+1))$. In this context, let $h_{1}(k)$ and $h_{2}(k)$ denote the channel coefficients at time index $k$ and $h_{1}(k+1) \doteq h_{1}(k)+\Delta_{1}(k)$ and $h_{2}(k+1) \doteq h_{2}(k)+\Delta_{2}(k)$ the ones at time index $k+1$. Accordingly, the channel matrix is given by

$$
\tilde{\mathbf{H}}=\left[\begin{array}{cc}
h_{1}(k) & -h_{2}(k) \\
h_{2}^{*}(k+1) & h_{1}^{*}(k+1)
\end{array}\right]
$$

i.e., (14) becomes

$$
\hat{\mathbf{x}}=\tilde{\mathbf{H}}^{H} \tilde{\mathbf{H}} \mathbf{x}(k)+\tilde{\mathbf{H}}^{H} \mathbf{n}(k)
$$

provided that the channel coefficients are perfectly known at the receiver both for time index $k$ and for time index $k+1$. The matrix $\tilde{\mathbf{H}}^{H} \tilde{\mathbf{H}}$ does not have a diagonal structure

$$
\begin{aligned}
& \tilde{\mathbf{H}}^{H} \tilde{\mathbf{H}} \\
& =\left[\begin{array}{cc}
\left|h_{1}(k)\right|^{2}+\left|h_{2}(k)+\Delta_{2}(k)\right|^{2} & \zeta^{*} \\
\zeta & \left|h_{1}(k)+\Delta_{1}(k)\right|^{2}+\left|h_{2}(k)\right|^{2}
\end{array}\right] \\
& \text { where } \zeta=h_{1}(k) \Delta_{2}^{*}(k)+h_{2}^{*}(k) \Delta_{1}(k)+\Delta_{1}(k) \Delta_{2}^{*}(k) .
\end{aligned}
$$

As in Section IV-B1, the desired symbols $x(k)$ and $x(k+1)$ are still combined in a constructive way but weighted with different factors. The secondary diagonal elements $\zeta$ and $\zeta^{*}$ introduce an additional error term to the estimates $\hat{x}(k)$ and $\hat{x}(k+1)$ solely due to the loss of orthogonality. Numerical results, however, indicate that this additional error term is negligible, even for high velocities of the MS. Note that for $\Delta_{1}(k), \Delta_{2}(k) \rightarrow 0$, (19) reduces to (14).

3) Orthogonality Loss Due to Frequency-Selective Fading: In the case of a quasi-static frequency-selective fading channel with memory length $L$, the two transmission paths from either transmit antenna to the receive antenna can be described by means of two complex-valued channel coefficient vectors $\mathbf{h}_{\mathbf{1}}=\left[h_{1}^{(0)}, h_{1}^{(1)}, \ldots, h_{1}^{(L)}\right]$ and $\mathbf{h}_{\mathbf{2}}=\left[h_{2}^{(0)}, h_{2}^{(1)}, \ldots, h_{2}^{(L)}\right]$. If a channel memory length of $L=1$ is considered, (13) becomes

$$
\mathbf{y}(k)=\mathbf{H}_{0} \mathbf{x}(k)+\mathbf{H}_{\mathrm{ISI}, 1} \mathbf{x}^{*}(k)+\mathbf{H}_{\mathrm{ISI}, 2} \mathbf{x}^{*}(k-2)+\mathbf{n}(k)
$$

where

$$
\mathbf{H}_{0}=\left[\begin{array}{cc}
h_{1}^{(0)} & -h_{2}^{(0)} \\
h_{2}^{(0) *} & h_{1}^{(0) *}
\end{array}\right] \quad \mathbf{H}_{\mathrm{ISI}, 1}=\left[\begin{array}{cc}
0 & 0 \\
h_{1}^{(1) *} & -h_{2}^{(1) *}
\end{array}\right]
$$

and

$$
\mathbf{H}_{\mathrm{ISI}, 2}=\left[\begin{array}{cc}
h_{2}^{(1)} & h_{1}^{(1)} \\
0 & 0
\end{array}\right] \text {. }
$$

The orthogonal properties of the Alamouti scheme are, therefore, lost in the presence of ISI (in this example, represented by

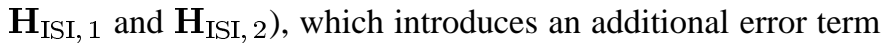
determined by the elements of $\mathbf{H}_{\mathrm{ISI}, 1}$ and $\mathbf{H}_{\mathrm{ISI}, 2}$. Note that for $h_{1}^{(1)}, h_{2}^{(1)}=0,(21)$ reduces to (13).

The performance of the Alamouti scheme on frequency-selective channels has also been studied in [15]. An extension of the classical scheme to channels with ISI has been proposed in [16].

\section{Simulation Results}

The simulation results presented here demonstrate the performance of the GSM/EDGE system enhanced by delay-diversity and the Alamouti scheme on a GSM 05.05 TU wireless channel [11], which is a frequency-selective channel with memory length of about $L_{\mathrm{TU}}=3$. In particular, the influence of a time-variant channel due to motion of the MS is considered as well as the influence of nonperfect knowledge of the channel coefficients at the receiver. In case of the Alamouti scheme, the application of different TS pairs is addressed. 
All simulation results presented in the following were obtained by means of Monte Carlo simulations over 10000 bursts. The simulations are restricted to binary GMSK modulation. Moreover, the transmission of uncoded data is regarded, i.e., channel coding is not performed. In the enhanced systems two transmit antennas and up to two receive antennas are deployed. In this context, the overall transmitter power is the same as for a single transmit antenna; i.e., the transmission power is normalized by a factor $1 / 2$ at each antenna. Normalization with respect to the number $n_{R}$ of receive antennas has not been performed. The receiver filter used is the nonadaptive root-raised-cosine filter introduced in Section IV-A2. The employed Max-Log-MAP detector has $2^{L_{\mathrm{eq}}}=2^{L_{\mathrm{TU}}}=8$ states in the case of the $(1 \times 1)$ system. In the system enhanced by delay diversity utilizing a delay $\delta, 2^{L_{\mathrm{TU}}+\vartheta}$ trellis states are required for optimal equalization and detection, where $\vartheta=\lfloor\delta / T\rfloor$ (as in general, $\delta \neq m \cdot T$ with $m$ being an integer number). In the Alamouti case, $2^{L_{\mathrm{TU}}+1}=2^{4}$ states are required, since $L_{\mathrm{TU}}$ is an odd number. Trellis termination has not been applied in the Alamouti case. Note that in the case of 8-PSK signaling, the complexity of the Max-Log-MAP detector will exceed limits of a practical implementation already for the $(1 \times 1)$ system $\left(8^{L_{\mathrm{TU}}}=512\right.$ trellis states $)$. Therefore, reduced-state techniques are mandatory here, for both the conventional and the enhanced systems.

\section{A. Performance of the Enhanced Systems on the TUO Channel}

The bit-error performance of the GSM/EDGE system enhanced by the delay-diversity and the Alamouti scheme on the time-invariant TU channel (TU0) is shown in Fig. 7(a) and (b). The channel coefficients are perfectly known at the receiver.

The BER curves are plotted as a function of the average SNR $E_{s} / N_{0}$. As a reference, the BER curve resulting for the $(1 \times 1)$ system is included. Moreover, analytical curves for diversity reception of uncoded BPSK are included [21, Ch. 14.4], where data symbols $x(k) \in\{ \pm 1\}$ are transmitted over $\nu$ individual paths subject to independent Rayleigh fading and characterized by an identical average SNR of $\left(E_{s} / N_{0}\right) / \nu$.

As shown in Fig. 7(a), the delay-diversity scheme yields significant performance improvements upon the $(1 \times 1)$ system. Deploying a delay $\delta=T$, the gain accomplished at a BER of $10^{-3}$ is about $3 \mathrm{~dB}$ when a single receive antenna is employed $[(2 \times 1)$ delay diversity $]$ and about $10 \mathrm{~dB}$ in the case of a second receive antenna $[(2 \times 2)$ delay diversity]. A delay $\delta=3 T$ even leads to a gain of $4.7 \mathrm{~dB}$ for $n_{R}=1$, which marks the maximum gain attainable by means of a single receive antenna (cf. Fig. 4). This gain comes, however, at the expense of an increased equalizer-detector complexity.

The Alamouti scheme accomplishes smaller gains than the delay-diversity scheme with $\delta=T$, namely, $1.5 \mathrm{~dB}[(2 \times 1)$ Alamouti] and $9 \mathrm{~dB}[(2 \times 2)$ Alamouti] in the presence of one and two receive antennas, respectively [cf. Fig. 7(b)]. It is worth noting here that-in contrast to the classical Alamouti scheme- the extended scheme proposed in [16] yields a better performance on the GSM 05.05 channels than delay diversity [17].

Fig. 8 illustrates the performance of delay diversity, when $n_{T}>2$ transmit antennas are employed in connection with a

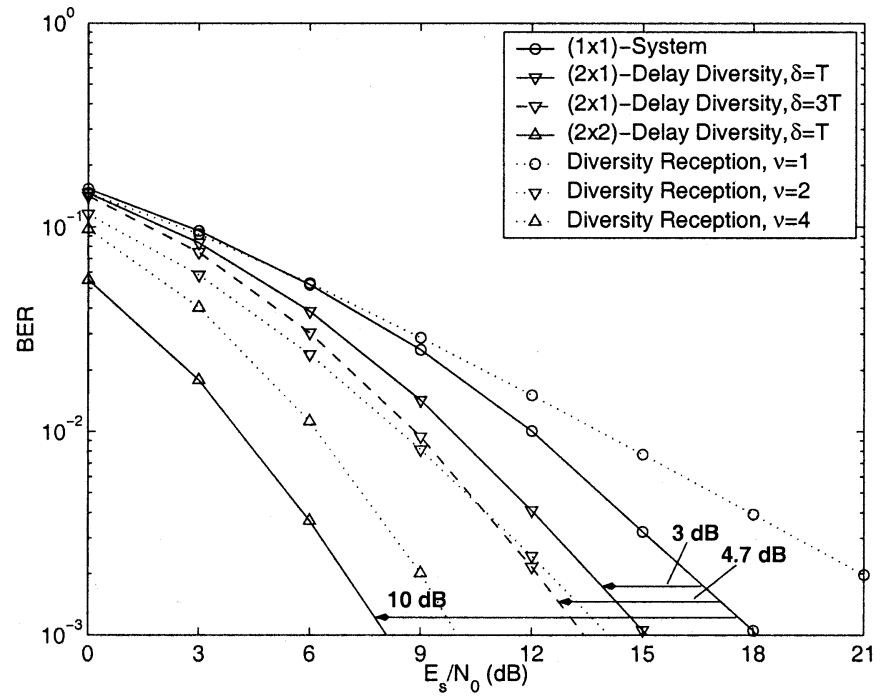

a)

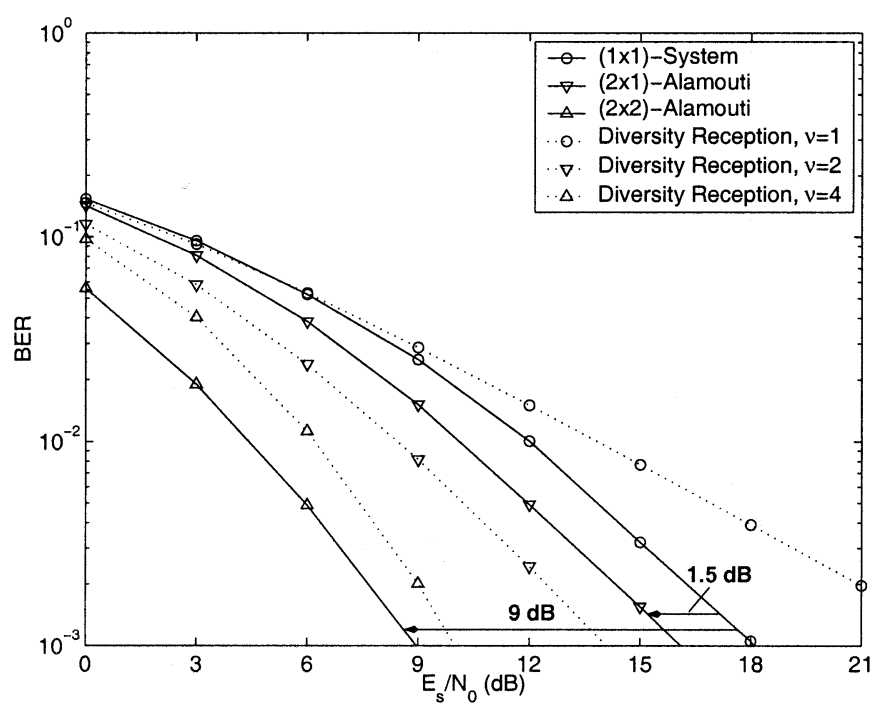

b)

Fig. 7. Performance on the TU0 channel, perfect knowledge of channel coefficients at the receiver. (a) Delay-diversity scheme. (b) Alamouti scheme.

delay $\delta_{i}=(i-1) T$ at antenna $i\left(1 \leq i \leq n_{T}\right.$, transmission power normalized by $1 / n_{T}$ at each antenna). The performance gains at a BER of $10^{-3}$ with respect to the $(1 \times 1)$ system are plotted as a function of the equalizer-detector complexity. The optimal equalizer-detector has $2^{L_{\mathrm{TU}}+n_{T}-1}$ states, i.e., the complexity grows exponentially with $n_{T}$. For comparison, the gains accomplished by means of $n_{T}=2$ antennas and delays $\delta>T$ are included in Fig. 8.

\section{B. Choice of Different TS Pairs in the Alamouti Case}

Fig. 9 demonstrates the special importance of an appropriate TS pair in the case of the Alamouti scheme $\left(n_{R}=1\right.$ receive antenna). Three different cases of TS pairs are considered, where each time GSM TS 1 is employed for the signal at the first transmit antenna [cf. Table I(A)]. In the first case, the optimized partner sequence with respect to GSM TS 1 is employed at the second transmit antenna [cf. Table I(B)]. In the second and the 


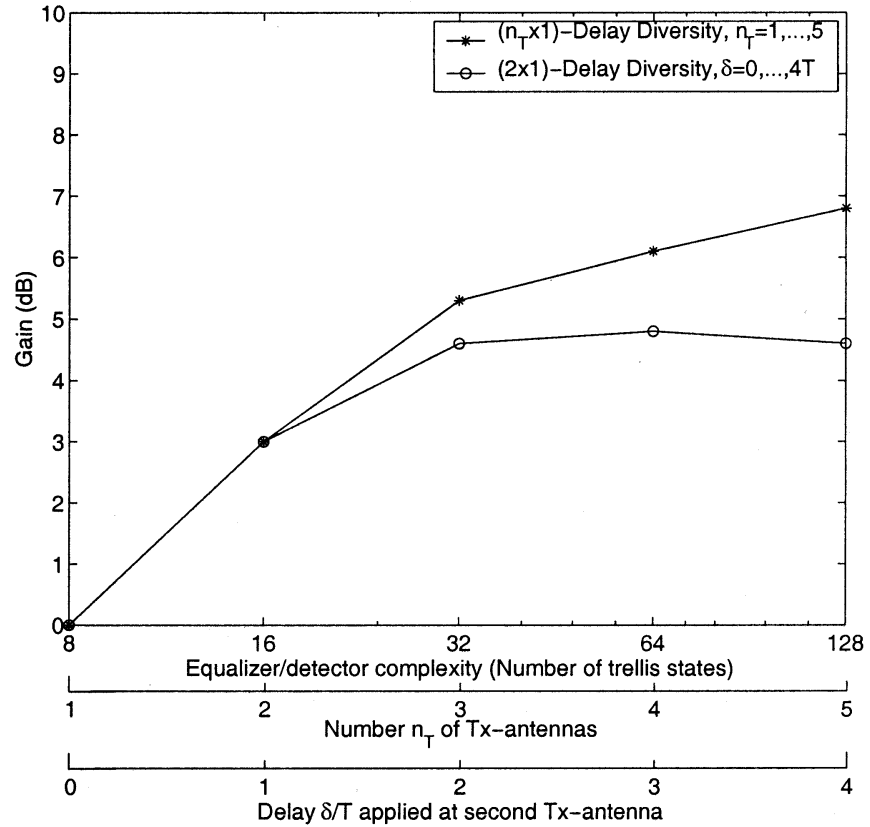

Fig. 8. Gain in $E_{s} / N_{0}$ with respect to the $(1 \times 1)$ system at a BER of $10^{-3}$, accomplished by means of (a) $n_{T}>2$ transmit antennas and a delay $\delta_{i}=$ $(i-1) T$ at antenna $i\left(1 \leq i \leq n_{T}\right)$ and (b) $n_{T}=2$ transmit antennas and a delay $\delta>T$ at the second antenna.

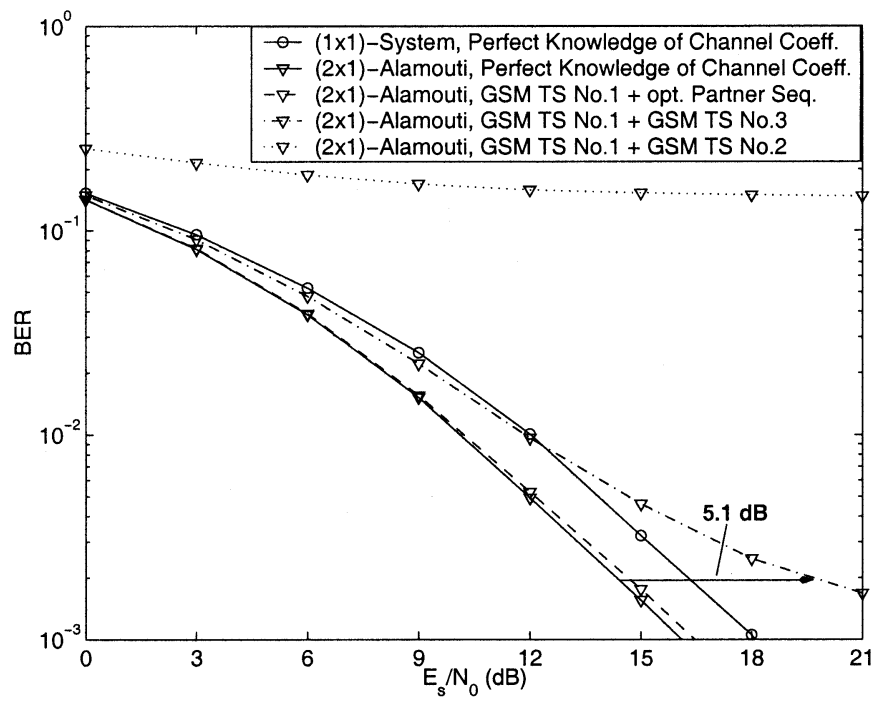

Fig. 9. Performance of the Alamouti scheme on the TU0 channel using different pairs of TSs [CE by means of correlation method (no AWGN)].

third case, the pairs (GSM TS $1+3)$ and (GSM TS $1+2)$ are considered. The channel coefficient estimates are obtained by means of the correlation method. It is important to note that the estimates are not corrupted by additional noise. The performance degradations shown in Fig. 9 are, therefore, solely due to nonperfect cross-correlation properties of the employed TS pairs.

The application of the optimized partner sequence leads to a very small performance deterioration with respect to perfect knowledge of the channel coefficients at the receiver, which is about $0.3 \mathrm{~dB}$ at a BER of $2 \cdot 10^{-3}$. The pair (GSM TS $1+3$ ) is the best choice among all possible pairs of GSM sequences in the sense of a minimum average CE error for large SNR values (TU-profile). Still, its application leads to a performance degradation of about $5.1 \mathrm{~dB}$ at the same BER. The optimized partner sequence of Table I(B), therefore, significantly outperforms any pair of GSM sequences. Note that for a desired BER of $<0.01$, the $(2 \times 1)$ Alamouti scheme employing the TS pair (GSM TS $1+3)$ is even less efficient than the $(1 \times 1)$ system. The pair $($ GSM TS $1+2)$ turns out to be a particularly bad choice and leads to further significant performance deterioration. In this case the $(2 \times 1)$ Alamouti scheme does not accomplish a BER $\leq 0.1$

\section{Influence of a Time-Variant Channel}

Fig. 10(a) and (b) shows the influence of a time-variant channel on the performance of the GSM/EDGE system enhanced by the delay-diversity $\left(n_{T}=2, \delta=T\right)$ and the Alamouti scheme (GSM TS 1 employed in connection with its optimized partner sequence). Considered are the time-variant TU channels TU3, TU50, and TU100 representing vehicular speeds of 3,50 , and $100 \mathrm{~km} / \mathrm{h}$, respectively, at a carrier frequency of $f_{c}=900 \mathrm{MHz}$. In all cases, the channel coefficient estimates are obtained by means of the correlation method and are not corrupted by additional noise. Within each burst they hold, however, only for the position of the midamble TS. Toward the edges of each burst, the current channel coefficients might significantly differ from the ones applying for the midamble, especially when the MS moves with a high speed.

As Fig. 10(a) and (b) shows, a time-variant channel causes only little degradation both in the case of delay diversity and in the case of the Alamouti scheme, as long as the MS moves with a speed $\leq 50 \mathrm{~km} / \mathrm{h}$. Generally, the enhanced systems become even more robust when a second receive antenna is employed.

\section{Influence of Nonperfect Knowledge of the Channel Coefficients}

Fig. 11(a) and (b) shows the influence of nonperfect knowledge of the channel coefficients at the receiver on the performance of the enhanced systems (TU0 channel); i.e., this time the channel coefficient estimates are corrupted by additional noise. In turn, the channel coefficient estimates are obtained by means of the correlation method. For the delay-diversity scheme the delay $\delta=T$ is considered. In the Alamouti case, the optimized partner sequence with respect to GSM TS 1 is employed.

In the case of delay diversity, at a BER of $10^{-3}$, a performance degradation of 1.1 and $1.3 \mathrm{~dB}$ occurs when one and two receive antennas are employed, respectively. For comparison, in the case of a $(1 \times 1)$ system, a degradation of about $1 \mathrm{~dB}$ can be expected.

Compared with delay diversity, the Alamouti scheme tends to be more sensitive to nonperfect knowledge of the channel coefficients. In the case $n_{R}=1$ a performance degradation of $1.7 \mathrm{~dB}$ results and in the case $n_{R}=2$ a degradation of $1.9 \mathrm{~dB}$.

\section{SUMMARY AND CONCLUSION}

The application of the delay-diversity and the Alamouti scheme in a GSM/EDGE system using two transmit and up to two receive antennas has been investigated. 


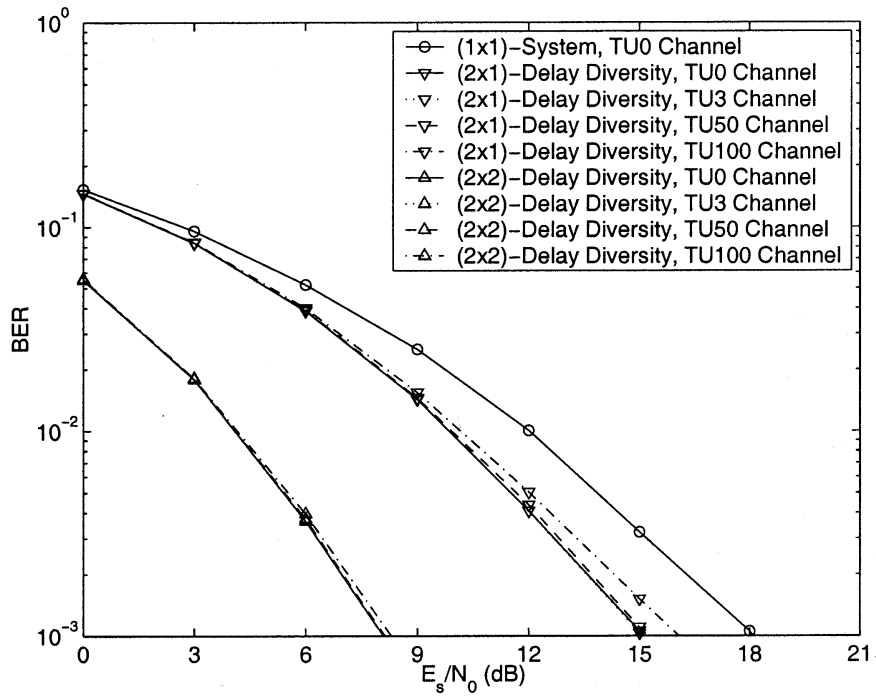

a)

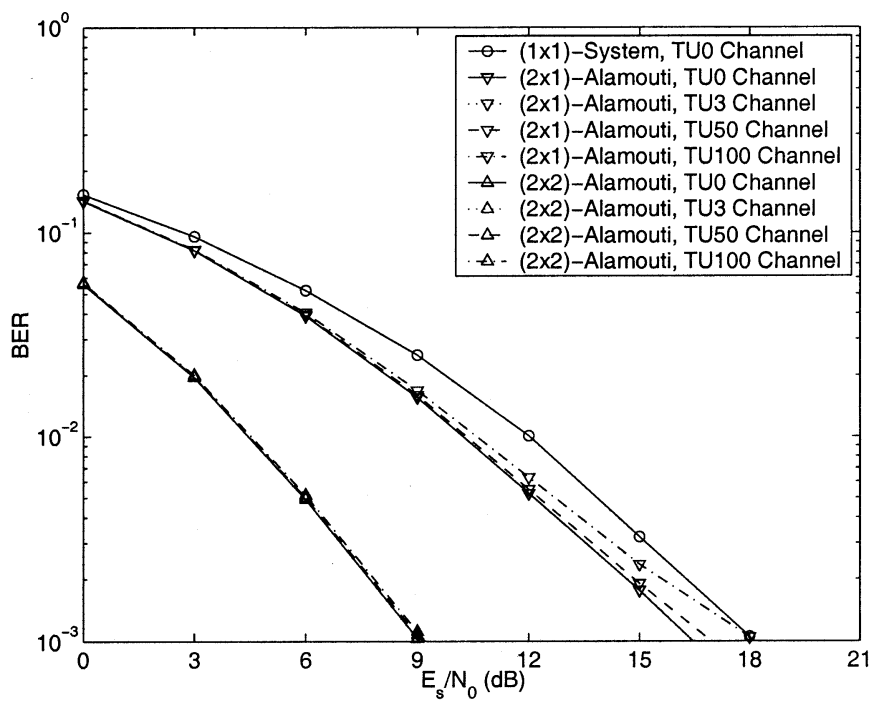

b)

Fig. 10. Influence of a time-variant TU channel [CE by means of correlation method (no AWGN)]. (a) Delay-diversity scheme $(\delta=T)$. (b) Alamouti scheme (GSM TS 1 + optimized partner).

First of all, the transmitter structures and the receiver structure were presented resulting in the GSM/EDGE system enhanced by either STC scheme. The transmitter structures are compatible with current specifications. For the case of the Alamouti scheme, a modified Max-Log-MAP equalization and detection algorithm was presented, which is of the same complexity as the conventional algorithm. Moreover, appropriate pairs of TSs were introduced, consisting of one of the eight GSM sequences and a corresponding optimized partner sequence. Simulation results for a TU environment showed that the proposed sequence pairs yield a system performance very close to the optimal case. In fact, they significantly outperform any pair of GSM sequences.

For the $(2 \times 1)$ delay-diversity scheme, an improved RRB was derived as a function of the delay $\delta$ applied to the signal at the second transmit antenna. Both the case of an optimal and the case of a suboptimal trellis-based equalizer-detector were con-

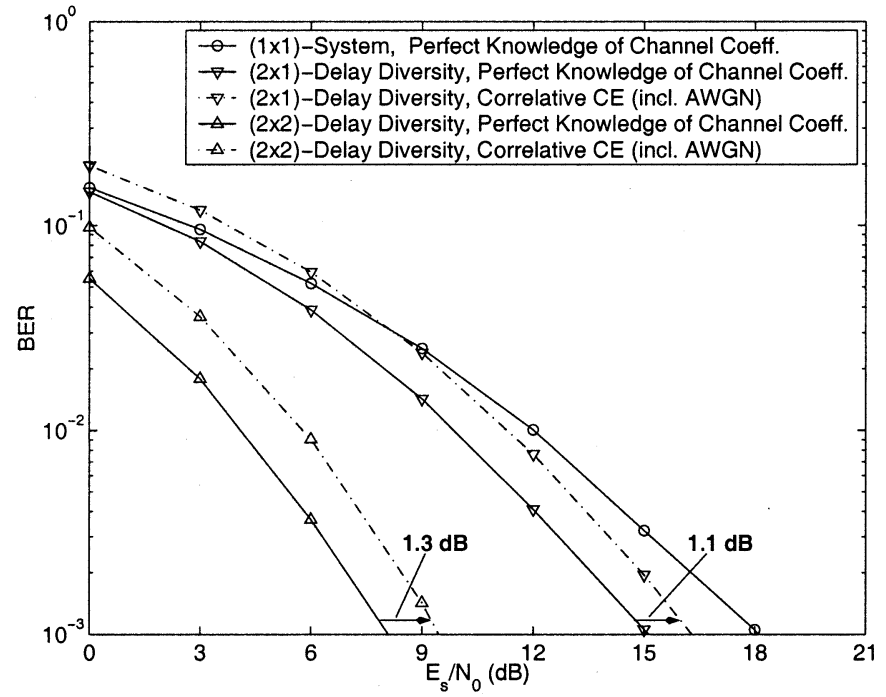

a)

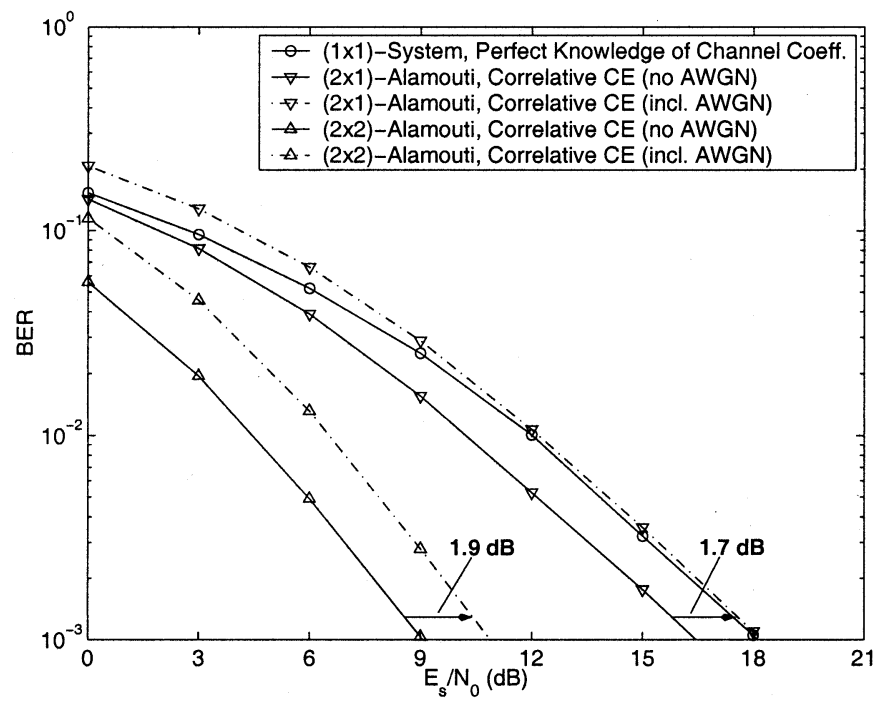

b)

Fig. 11. Influence of nonperfect knowledge of the channel coefficients (TU0 channel, CE by means of correlation method). (a) Delay-diversity scheme ( $\delta=$ $T$ ). (b) Alamouti scheme (GSM TS $1+$ optimized partner).

sidered. In particular, the influence of a sampling phase $0 \leq \epsilon<$ $T$ and the influence of the average SNR was addressed. Finally, a rule-of-thumb was deduced from the RRB concerning the optimal choice of $\delta$. It was shown that the conventional choice $\delta=T$ is not necessarily the best one.

For the Alamouti scheme, it was shown that its orthogonal properties are lost in the case of nonperfect knowledge of the channel coefficients at the receiver as well as in the presence of fast and/or frequency-selective fading. This loss of orthogonality results in a performance degradation.

On the basis of simulation results for a TU wireless channel, it was demonstrated that both the delay-diversity and the Alamouti scheme yield significant performance improvements upon a single transmit antenna system. They are, therefore, attractive techniques for an extension of the current GSM/EDGE specifications with regard to future wireless services requiring high 
bit rates. With a single receive antenna, performance improvements up to $3 \mathrm{~dB}$ were accomplished at a BER of $10^{-3}$, with respect to the average SNR $E_{s} / N_{0}$. Utilization of a second receive antenna yielded even larger performance improvements (up to $10 \mathrm{~dB}$ ), due to the fact that the number of independent signal paths is doubled.

With regard to a time-variant TU channel, it was shown that only small performance degradations result for both STC schemes, as long as the MS does not move significantly faster than $50 \mathrm{~km} / \mathrm{h}$ (at a carrier frequency of $900 \mathrm{MHz}$ ).

Finally, the influence of nonperfect knowledge of the channel coefficients at the receiver was considered. For the case of delay diversity, it was shown that the performance degradation is comparable with that occurring in a $(1 \times 1)$ system $(\approx 1 \mathrm{~dB}$ at a BER of $10^{-3}$ ). In the case of the Alamouti scheme, however, the performance degradation tended to be slightly higher.

Generally, the simulation results presented here indicate that delay diversity is more efficient than the Alamouti scheme. First of all, delay diversity outperforms the Alamouti scheme on the $\mathrm{TU}$ wireless channel, both in the case of one and two receive antenna(s). Second, delay diversity tends to be less sensitive to nonperfect knowledge of the channel coefficients at the receiver. Another advantage of delay diversity is the fact that virtually the same equalization and detection algorithm can be used for the detection of the transmitted symbols as is employed in the $(1 \times 1)$ system, whereas in the case of the Alamouti scheme, a modified algorithm is required.

\section{APPENDIX}

\section{A. Elimination of the Static ISI}

A convolution of the received symbols $y(k)$ according to (5) with the ZF equalizer yields

$$
\begin{aligned}
y(k) & * e_{\mathrm{ZF}}^{(\epsilon)}(k) \\
= & \left(\sum_{l=0}^{L} h^{(l)}(k, \delta) x(k-l)\right) * e_{\mathrm{ZF}}^{(\epsilon)}(k)+\overbrace{n(k) * e_{\mathrm{ZF}}^{(\epsilon)}(k)}^{\tilde{n}(k)} \\
= & \sum_{l=0}^{L} \underbrace{\left(\sum_{l^{\prime}=0}^{L} h^{\left(l^{\prime}\right)}(k, \delta) e_{\mathrm{ZF}}^{(\epsilon)}\left(l-l^{\prime}\right)\right)}_{h^{(l)}(k, \delta) * e_{\mathrm{ZF}}^{(\epsilon)}(l) \doteq \tilde{h}^{(l)}(k, \delta)} x(k-l)+\tilde{n}(k) \\
= & \sum_{l=0}^{L} \tilde{h}^{(l)}(k, \delta) x(k-l)+\tilde{n}(k) .
\end{aligned}
$$

In turn, the derivation of $\tilde{\rho}_{l} \doteq E\left\{\left|\tilde{h}^{(l)}(k, \delta)\right|^{2}\right\}=$ $E\left\{\left|h^{(l)}(k, \delta) * e_{\mathrm{ZF}}^{(\epsilon)}(l)\right|^{2}\right\}$ is essentially done as in $[26$, Appendix II], leading to (9). Note that the colored noise samples $\tilde{n}(k)$ do not affect the analysis.

\section{B. Transformed SNR Value for the Modified Channel Model}

Consider a channel model characterized by a channel memory length $L$, which in the following shall be transformed into a modified channel model of memory length $K<L$. The received signal $y(k)$ can be split into two terms

$$
\begin{aligned}
y(k) & =\sum_{l=0}^{L} h^{(l)}(k) x(k-l)+n(k) \\
& =\sum_{l=0}^{K} h^{(l)}(k) x(k-l)+\overbrace{\underbrace{\sum_{l=K+1}^{L} h^{(l)}(k) x(k-l)}_{\doteq n^{\prime}(k)}+n(k)}^{\doteq y_{\mathrm{ISI}}(k)} .
\end{aligned}
$$

The noise term $n^{\prime}(k)$ comprises an ISI expression $y_{\mathrm{ISI}}(k)$, which is due to the neglect of the channel coefficients $\left[h^{(K+1)}(k), \ldots, h^{(L)}(k)\right]$ in the modified channel model. For the corresponding mean noise power $\left(N_{0} / E_{s}\right)^{\prime}$, one obtains

$$
\begin{aligned}
\left(\frac{N_{0}}{E_{s}}\right)^{\prime} & =E\left\{\left|n^{\prime}(k)\right|^{2}\right\}=E\left\{\left|y_{\mathrm{ISI}}(k)+n(k)\right|^{2}\right\} \\
& \stackrel{(*)}{=} E\left\{\left|y_{\mathrm{ISI}}(k)\right|^{2}\right\}+E\left\{|n(k)|^{2}\right\} \\
& =\sum_{l=K+1}^{L} \underbrace{E\left\{\left|h^{(l)}(k)\right|^{2}\right\}}_{=\rho_{l}}+\frac{N_{0}}{E_{s}}
\end{aligned}
$$

where a binary modulation scheme is assumed in conjunction with independent and identically distributed (i.i.d.) data symbols $x(k)$; i.e., $E\left\{|x(k)|^{2}\right\}=1$. Step $(*)$ is permitted under the assumptions that $y_{\mathrm{ISI}}(k)$ and $n(k)$ are statistically independent and that $E\{n(k)\}$ and $E\left\{y_{\mathrm{ISI}}(k)\right\}$ are zero. The latter assumption is granted due to the i.i.d. data symbols.

\section{ACKNOWLEDGMENT}

The authors would like to thank Dr. P. Strauch for fruitful discussions and the anonymous reviewers for their valuable suggestions.

\section{REFERENCES}

[1] G. J. Foschini, "Layered space-time architecture for wireless communication in a fading environment when using multi-element antennas," Bell Syst. Tech. J., pp. 41-59, Autumn 1996.

[2] V. Tarokh, N. Seshadri, and A. R. Calderbank, "Space-time codes for high data rate wireless communication: Performance criterion and code construction," IEEE Trans. Inform. Theory, vol. 44, pp. 744-765, Mar. 1998.

[3] V. Tarokh, A. Naguib, N. Seshadri, and A. R. Calderbank, "Space-time codes for high data rate wireless communication: Performance criteria in the presence of channel estimation errors, mobility, and multiple paths," IEEE Trans. Commun., vol. 47, pp. 199-207, Feb. 1999.

[4] S. Bäro, G. Bauch, and A. Hansmann, "Improved codes for space-time trellis coded modulation," IEEE Commun. Lett., vol. 4, pp. 20-22, Jan. 2000.

[5] Y. Gong and K. B. Letaief, "Performance evaluation and analysis of space-time coding for high data rate wireless personal communications," in Proc. IEEE VTC Fall'99, 1999, pp. 1331-1335.

[6] V. Tarokh, H. Jafarkhani, and A. R. Calderbank, "Space-time block coding for wireless communications: Performance results," IEEE J. Select. Areas Commun., vol. 17, no. 3, pp. 451-460, Mar. 1999.

[7] - "Space-time block codes from orthogonal designs," IEEE Trans. Inform. Theory, vol. 45, pp. 1456-1467, June 1999.

[8] A. Wittneben, "A new bandwidth efficient transmit antenna modulation diversity scheme for linear digital modulation," in Proc. IEEE ICC, 1993, pp. 1630-1634. 
[9] N. Seshadri and J. H. Winters, "Two signaling schemes for improving the error performance of frequency-division-duplex (FDD) transmission systems using transmitter antenna diversity," Int. J. Wireless Inform. Networks, vol. 1, no. 1, pp. 49-59, 1994.

[10] S. M. Alamouti, "A simple transmit diversity technique for wireless communications," IEEE J. Select. Areas Commun., vol. 16, pp. $1451-1458$, Oct. 1998

[11] "Digital cellular communications system (Phase 2+)," ETSI/3GPP, Sophia-Antipolis, France, Technical Specifications 3GPP TS 05.01-05, 2001.

[12] P. Robertson, P. Hoeher, and E. Villebrun, "Optimal and sub-optimal maximum a posteriori algorithms suitable for turbo decoding," Eur. Trans. Telecommun., vol. 8, pp. 119-125, Mar./Apr. 1997.

[13] M. Coupechoux and V. Braun, "Space-time coding for the EDGE mobile radio system," in Proc. IEEE Int. Conf. Pers. Wireless Commun., 2000, pp. 28-32.

[14] R. Srinivasan, M. J. Heikkila, and R. Pirhonen, "Performance evaluation of space-time coding for EDGE," in Proc. IEEE ICC, 2001, pp. 3056-3060.

[15] H. Boelcskei and A. J. Paulraj, "Space-frequency coded broadband OFDM systems," in Proc. WCNC, 2000, pp. 1-6.

[16] E. Lindskog and A. Paulraj, "A transmit diversity scheme for channels with intersymbol interference," in Proc. IEEE ICC, 2000, pp. 307-311.

[17] D. Flore and E. Lindskog, "Time reversal space-time block coding vs. transmit delay diversity-A comparison based on a GSM-like system," in Proc. 9th DSP Workshop, Hunt, TX, 2000.

[18] K. Zangi, D. Hui, and J.-F. Cheng, "Physical layer issues for deploying transmit diversity in GPRS/EGPRS networks," in Proc. IEEE VTC Fall 2001 , pp. 538-542.

[19] C. Budianu and L. Tong, "Channel estimation for space-time orthogonal block codes," in Proc. IEEE ICC, 2001, pp. 1127-1131.

[20] W. J. Choi and J. M. Cioffi, "Multiple input/multiple output (MIMO) equalization for space-time block coding," in Proc. IEEE Pacific Rim Conf. Commun., 1999, pp. 341-344.

[21] J. G. Proakis, Digital Communications, 4th ed. New York: McGrawHill, 2001.

[22] A. F. Naguib, "On the matched filter bound of transmit diversity techniques," in Proc. IEEE ICC, 2001, pp. 596-603.

[23] A. Baier, "Correlative and iterative channel estimation in adaptive Viterbi equalizers for TDMA mobile radio systems," in ITG-Fachbericht 107, 1989, pp. 363-368.

[24] G. Caire and U. Mitra, "Structured multiuser channel estimation for block-synchronous DS/CDMA," IEEE Trans. Commun., vol. 49, pp. $1605-1617$, Sept. 2001.

[25] L. R. Bahl, J. Cocke, F. Jelinek, and J. Raviv, "Optimal decoding of linear codes for minimizing symbol error rate," IEEE Trans. Inform. Theory, vol. IT-20, pp. 284-287, Mar. 1974.

[26] P. Hoeher and S. Badri, "On the timing sensitivity of symbol-spaced trellis-based equalizers applied to frequency-selective fading channels," in Proc. IEEE GLOBECOM'98, 1998, pp. 88-93.

[27] G. Bauch, "Turbo-Entzerrung' und Sendeantennen-Diversity mit 'Space-Time-Codes' im Mobilfunk," Ph.D. dissertation (in German), Dept. Commun. Eng., Munich Univ. Technol., Munich, Germany, 2001.

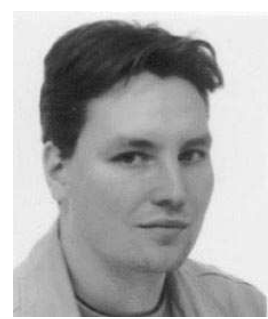

Jan Mietzner (S'02) was born in Rendsburg, Germany, in 1975. He received the Dipl.-Ing. degree in electrical engineering from the University of Kiel, Kiel, Germany, in 2001.

Since August 2001, he has been a Research Assistant at the Information and Coding Theory Lab, University of Kiel. His research interests concern physical layer aspects of future wireless communications systems as channel coding and especially space-time codes

Mr. Mietzner received the Prof. Dr. Werner Petersen-Award for his diploma thesis on space-time codes.

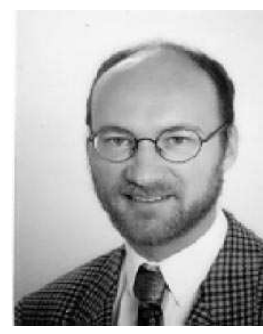

Peter A. Hoeher (M'89-SM'97) was born in Cologne, Germany, in 1962. He received the Dipl.-Eng. and Dr.-Eng. degrees in electrical engineering from the Technical University of Aachen, Aachen, Germany, in 1986, and the University of Kaiserslautern, Kaiserslautern, Germany, in 1990.

From October 1986 to September 1998, he was with the German Aerospace Center (DLR), Oberpfaffenhofen, Germany. From December 1991 to November 1992, he was on leave at AT\&T Bell Laboratories, Murray Hill, NJ. In October 1998, he joined the University of Kiel, Kiel, Germany, where he is currently a Professor in Electrical Engineering. His research interests are in the general area of communication theory with applications in wireless communications, including digital modulation techniques, channel coding, iterative processing, equalization, multiuser detection, and channel estimation.

Dr. Hoeher received the Hugo-Denkmeier-Award in 1990. He currently serves as an Associated Editor for the IEEE TRANSACTIONS ON COMMUNICATIONS

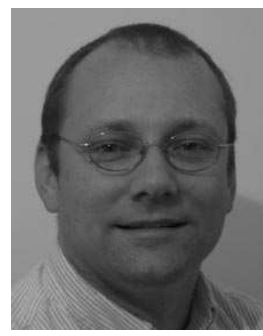

Magnus Sandell (S'92-A'97) received the M.Sc. degree in electrical engineering and the Ph.D. degree in signal processing from the Luleå University of Technology, Luleå, Sweden, in 1990 and 1996, respectively.

He spent six months as a Researcher with the Division of Signal Processing, Luleå University of Technology, and then joined Bell Laboratories, Lucent Technologies, Swindon, U.K., in 1997. Since March 2002, he has worked for Toshiba Research Europe, Bristol, U.K., in the Telecommunications Research Laboratory. His research interests include signal processing and digital communications theory. Currently his focus is on multiple antenna systems and space-time coding. 